\title{
Identification, Classifications, and Absolute Properties of 773 Eclipsing Binaries Found in the Trans-atlantic Exoplanet Survey
}

\section{Citation}

Devor, Jonathan, David Charbonneau, Francis T. O'Donovan, Georgi Mandushev, and Guillermo Torres. 2008. "IDENTIFICATION, CLASSIFICATIONS, AND ABSOLUTE PROPERTIES OF 773 ECLIPSING BINARIES FOUND IN THE TRANS-ATLANTIC EXOPLANET SURVEY." The Astronomical Journal 135 (3): 850-77. https://doi.org/10.1088/0004-6256/135/3/850.

\section{Permanent link}

http://nrs.harvard.edu/urn-3:HUL.InstRepos:41417347

\section{Terms of Use}

This article was downloaded from Harvard University's DASH repository, and is made available under the terms and conditions applicable to Other Posted Material, as set forth at http:// nrs.harvard.edu/urn-3:HUL.InstRepos:dash.current.terms-of-use\#LAA

\section{Share Your Story}

The Harvard community has made this article openly available.

Please share how this access benefits you. Submit a story.

\section{Accessibility}




\title{
IDENTIFICATION, CLASSIFICATIONS, AND ABSOLUTE PROPERTIES OF 773 ECLIPSING BINARIES FOUND IN THE TRANS-ATLANTIC EXOPLANET SURVEY
}

\author{
Jonathan Devor ${ }^{1}$, David Charbonneau ${ }^{1,4}$, Francis T. O’Donovan $^{2}$, Georgi Mandushev $^{3}$, and Guillermo Torres ${ }^{1}$ \\ ${ }^{1}$ Harvard-Smithsonian Center for Astrophysics, 60 Garden Street, Cambridge, MA 02138, USA; jdevor@ cfa.harvard.edu \\ 2 California Institute of Technology, 1200 East California Boulevard, Pasadena, CA 91125, USA \\ ${ }^{3}$ Lowell Observatory, 1400 West Mars Hill Road, Flagstaff, AZ 86001, USA \\ Received 2007 September 5; accepted 2007 December 5; published 2008 February 5
}

\begin{abstract}
In recent years, we have witnessed an explosion of photometric time-series data, collected for the purpose of finding a small number of rare sources, such as transiting extrasolar planets and gravitational microlenses. Once combed, these data are often set aside, and are not further searched for the many other variable sources that they undoubtedly contain. To this end, we describe a pipeline that is designed to systematically analyze such data, while requiring minimal user interaction. We ran our pipeline on a subset of the Trans-Atlantic Exoplanet Survey dataset, and used it to identify and model 773 eclipsing binary systems. For each system we conducted a joint analysis of its light curve, colors, and theoretical isochrones. This analysis provided us with estimates of the binary's absolute physical properties, including the masses and ages of their stellar components, as well as their physical separations and distances. We identified three types of eclipsing binaries that are of particular interest and merit further observations. The first category includes 11 low-mass candidates, which may assist current efforts to explain the discrepancies between the observation and the models of stars at the bottom of the main sequence. The other two categories include 34 binaries with eccentric orbits, and 20 binaries with abnormal light curves. Finally, this uniform catalog enabled us to identify a number of relations that provide further constraints on binary population models and tidal circularization theory.
\end{abstract}

Key words: binaries: eclipsing - catalogs - methods: data analysis - stars: statistics - techniques: photometric

Online-only material: color figures, machine-readable table

\section{INTRODUCTION}

Since the mid-1990s, there has been an explosion of largescale photometric variability surveys. The search for gravitational microlensing events, which were predicted by Paczynski (1986), motivated the first wave of surveys (e.g., OGLE: Udalski et al. 1994; EROS: Beaulieu et al. 1995; DUO: Alard \& Guibert 1997; MACHO: Alcock et al. 1998). Encouraged by their success, additional surveys, searching for gamma-ray bursts (e.g., ROTSE: Akerlof et al. 2000) and general photometric variabilities (e.g., ASAS: Pojmanski 1997) soon followed.

Shortly thereafter, with the discovery of the first transiting extrasolar planet (Charbonneau et al. 2000; Henry et al. 2000; Mazeh et al. 2000), a second wave of photometric surveys ensued (e.g., OGLE-III: Udalski 2003; TrES: Alonso et al. 2004; HAT: Bakos et al. 2004; SuperWASP: Christian et al. 2006; XO: McCullough et al. 2006; for a review, see Charbonneau et al. 2007). Each of these projects involved intensive efforts to locate a few proverbial "needles" hidden in a very large data haystack. With few exceptions, once the needles were found, thus fulfilling the survey's original purpose, the many gigabytes of photometric light curves (LCs) collected were not made use of in any other way. In this paper, we demonstrate how one can extract a great deal more information from these survey datasets, with comparably little additional effort, using automated pipelines. To this end, we have made all the software tools described in this paper freely available (see Web links to the source code and working examples), and they are designed to be used with any LC dataset.

\footnotetext{
4 Alfred P. Sloan Research Fellow.
}

In the upcoming decade, a third wave of ultra-large groundbased synoptic surveys (e.g., Pan-STARRS: Kaiser et al. 2002; LSST: Tyson 2002), and ultra-sensitive space-based surveys (e.g., KEPLER: Borucki et al. 1997; COROT: Baglin \& The COROT Team 1998; GAIA: Gilmore et al. 1998) are expected to come online. These surveys are designed to produce photometric datasets that will dwarf all preceding efforts. To make any efficient use of such large quantities of data, it will become imperative to have in place a large infrastructure of automated pipelines for performing even the most casual data mining query.

In this paper, we focus exclusively on the identification and analysis of eclipsing binary (EB) systems. EBs provide favorable targets, as they are abundant and can be well modeled using existing modeling programs (e.g., WD: Wilson \& Devinney 1971; EBOP: Popper \& Etzel 1981). Once modeled, EBs can provide a wealth of useful astrophysical information, including constraints on binary component mass distributions, mass-radius-luminosity relations, and theories describing tidal circularization and synchronization. These findings, in turn, will likely have a direct impact on our understanding of star formation, stellar structure, and stellar dynamics. These physical distributions of close binaries may even help solve open questions relating to the progenitors of Type Ia supernovae (Iben \& Tutukov 1984). In additional to these, EBs can be used as tools; both as distance indicators (Stebbing 1910; Paczynski 1997) and as sensitive detectors for tertiary companions via eclipse timing (Deeg et al. 2000; Holman \& Murray 2005; Agol et al. 2005).

In order to transform such large quantities of data into useful information, one must construct a robust and computationally efficient automated pipeline. Each step along the pipeline 
Table 1

Observational Parameters of the TrES Fields

\begin{tabular}{|c|c|c|c|c|c|c|c|}
\hline Field & Constellation & $\begin{array}{c}\alpha \\
(\mathrm{J} 2000)^{\mathrm{a}}\end{array}$ & $\begin{array}{c}\delta \\
(\mathrm{J} 2000)\end{array}$ & $\begin{array}{c}\text { Galactic } \\
\text { Coordinates }(1, b)\end{array}$ & $\begin{array}{c}\text { Starting } \\
\text { Epoch (HJD) }\end{array}$ & $\begin{array}{c}\text { Ending } \\
\text { Epoch (HJD) }\end{array}$ & $\begin{array}{c}\text { Duration } \\
\text { (days) }\end{array}$ \\
\hline And0 & Andromeda & 010930.1255 & +471430.453 & $(126.11,-015.52)$ & 2452878.9 & 2452934.9 & 56.0 \\
\hline Cas0 & Cassiopeia & 003909.8941 & +492116.519 & $(120.88,-013.47)$ & 2453250.8 & 2453304.6 & 53.8 \\
\hline $\mathrm{CrB} 0$ & Corona Borealis & 160102.6616 & +331812.634 & $(053.49,+048.92)$ & 2453493.8 & 2453536.8 & 43.0 \\
\hline Cyg1 & Cygnus & 200121.5633 & +500616.902 & $(084.49,+010.28)$ & 2453170.7 & 2453250.0 & 79.3 \\
\hline Dra0 & Draco & 164517.8177 & +564654.686 & $(085.68,+039.53)$ & 2453093.8 & 2453163.0 & 69.2 \\
\hline Her0 & Hercules & 164914.2185 & +455859.963 & $(071.61,+039.96)$ & 2452769.9 & 2452822.0 & 52.1 \\
\hline Lyr1 & Lyra & 190126.3713 & +465605.325 & $(077.15,+017.86)$ & 2453541.8 & 2453616.7 & 74.9 \\
\hline Per1 & Perseus & 034107.8581 & +373448.712 & $(156.37,-014.04)$ & 2453312.8 & 2453402.8 & 90.0 \\
\hline Tau0 & Taurus & 042021.2157 & +272102.713 & $(169.83,-015.94)$ & 2453702.7 & 2453770.9 & 68.2 \\
\hline UMa0 & Ursa Major & 095206.3560 & +540351.596 & $(160.87,+047.70)$ & 2453402.9 & 2453487.8 & 84.9 \\
\hline
\end{tabular}

Note. ${ }^{a}$ ICRS 2000.0 coordinates of the guide star, which is located at the center of the field of view.

Table 2

The Number of Sources and Yield of the TrES Fields

\begin{tabular}{lcccccc}
\hline \hline Field & $\begin{array}{c}\text { Number } \\
\text { of LCs }\end{array}$ & $\begin{array}{c}\text { Number of Observations } \\
\text { in Each LC }\end{array}$ & $\begin{array}{c}\text { Fraction (\%) } \\
\text { RMS }<1 \%\end{array}$ & $\begin{array}{c}\text { Fraction (\%) } \\
\text { RMS }<2 \%\end{array}$ & $\begin{array}{c}\text { Found } \\
\text { EBs }\end{array}$ & $\begin{array}{c}\text { EB Discovery } \\
\text { Yield (\%) }\end{array}$ \\
\hline And0 & 26495 & 2357 & 16.5 & 40.4 & 111 & 0.42 \\
Cas0 & 22615 & 2069 & 11.0 & 38.2 & 119 & 0.53 \\
CrB0 & 18954 & 1287 & 11.0 & 22.4 & 28 & 0.15 \\
Cyg1 & 17439 & 3256 & 30.3 & 65.7 & 125 & 0.72 \\
Dra0 & 15227 & 2000 & 11.8 & 26.4 & 42 & 0.28 \\
Her0 & 15916 & 974 & 16.8 & 35.0 & 28 & 0.18 \\
Lyr1 & 22964 & 2815 & 19.4 & 49.0 & 135 & 0.59 \\
Per1 & 20988 & 1647 & 15.9 & 38.4 & 93 & 0.44 \\
Tau0 & 14442 & 1171 & 13.1 & 32.5 & 68 & 0.47 \\
UMa0 & 10405 & 1343 & 13.6 & 29.5 & 24 & 0.23 \\
\hline
\end{tabular}

will either measure some property of the LC, or filter out LCs that do not belong, so as to reduce the congestion in the following, more computationally intensive steps. One can achieve substantial gains in speed by dividing the data into subsets, and processing them in parallel on multiple CPUs. The bottlenecks of the analysis are the steps that require user interaction. In our pipeline, we reduce user interaction to essentially yes/no decisions regarding the success of the EB models, and eliminate any need for interaction in all but two stages. We feel that this level of interaction provides good quality control, while minimizing its detrimental subjective effects.

The data that we analyzed originate from ten fields of the Trans-atlantic Exoplanet Survey (TrES; Alonso et al. 2004). TrES employs a network of three automated telescopes to survey $6^{\circ} \times 6^{\circ}$ fields-of-view. To avoid potential systematic noise, we use the data from only one telescope, Sleuth, located at the Palomar Observatory in Southern California (O'Donovan et al. 2004). This telescope has a $10 \mathrm{~cm}$ physical aperture and a photometric aperture of radius of $30^{\prime \prime}$. The number of LCs in each field ranges from 10,405 to 26,495 (see Table 1), for a total of 185,445 LCs. The LCs consist of $\sim 2000 r$-band photometric measurements at a 9 min cadence. These measurements were created by binning the image-subtraction results of five consecutive $90 \mathrm{~s}$ observations, thus improving their non-systematic photometric noise. As a result $\sim 16 \%$ of the LCs have an RMS $<1 \%$, and $\sim 38 \%$ of the LCs have an RMS $<2 \%$ (see Table 2). The calibration of TrES images, identification of stars therein, extraction, and decorrelation of the LCs is described elsewhere (Dunham et al. 2004; Mandushev et al. 2005; O'Donovan et al. 2006, 2007). TrES is currently an active survey that is continuously observing new fields, though for this paper we have limited ourselves to these ten fields.

\section{METHOD}

The pipeline we have developed is an extended version of the pipeline described by Devor (2005). At the heart of this analysis lie two computational routines that we have described in earlier papers: the Detached Eclipsing Binary Light curve fitter ${ }^{5}$ (DEBiL; Devor 2005), and the Method for Eclipsing Component Identification $^{6}$ (MECI; Devor \& Charbonneau 2006a, 2006b). DEBiL fits each LC to a geometric model of a detached EB (steps 3 and 5 below). This model consists of two luminous, limb-darkened spheres that orbit in a Newtonian two-body orbit. MECI restricts the DEBiL fit along theoretical isochrones, and is thus able to create a physical model of each EB (step 9). This second model describes the masses and absolute magnitudes of the EB's stellar components, which are then used to determine the EB's distance and absolute separation.

The pipeline consists of ten steps. We elaborate on each of these steps below.

1. Determine the period.

2. If a distinct secondary eclipse is not observed, add an entry with twice the period.

3. Fit the orbital parameters with DEBiL.

4. Fine-tune the period using eclipse timing.

5. Refine the orbital parameters with DEBiL using the revised period.

6. Remove contaminated LCs.

7. Visually assess the quality of the EB models.

\footnotetext{
5 The DEBiL source code, utilities, and running example files are available online at http://www.cfa.harvard.edu/ jdevor/DEBiL.html.

6 The MECI source code and running examples are available online at http://www.cfa.harvard.edu/ jdevor/MECI.html.
} 


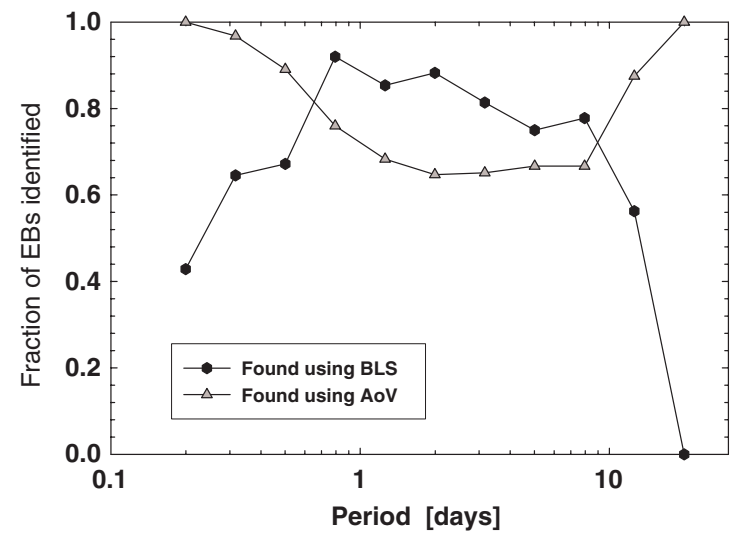

Figure 1. The fraction of the EBs in the final catalog found using the BLS algorithm and the AoV algorithm. The number of EBs in each bin is shown in Figure 8. The BLS method excelled at identifying EBs with short-duration eclipses (compared to the orbital period), which predominately occur at periods $>0.75$ days. The AoV method fared better with EBs that have long-duration eclipses, which predominately occur in sub-day periods. The AoV method also does well with EBs with period longer than 10 days, which may be dominated by giant-giant binaries (Derekas et al. 2007), and so also have broad eclipses. This plot demonstrates the importance of using multiple independent methods of identifying EB, otherwise the results will have a significant selection effect that may bias any statistical results.

8. Match the LC sources with external databases.

9. Estimate the absolute physical properties of the binary components using MECI.

10. Classify the resulting systems using both automatic and manual criteria.

We use the same filtering criteria as described in Devor (2005), both for removing LCs that are not periodic (step 1) and then for removing non-EB LCs (step 3). Together, these automated filters remove approximately $97 \%$ of the input LCs. In addition to these filters, we perform stringent manual inspections (steps 7 and 10) whereby we removed all the LCs we were not confident were EBs. These inspections ultimately removed approximately $86 \%$ of the remaining LCs. Thus only 1 out of every 240 input LCs were included in the final catalog.

In step 1, we use both the Box-fitting Least-Squares (BLS) period finder (Kovács et al. 2002), and a version of the analysis of variances (AoV) period finder (Schwarzenberg-Czerny 1989, 1996) to identify the periodic LCs within the dataset and to measure their periods. In our AoV implementation, we scan periods from 0.1 days up to the duration of each LC. We then select the period that minimizes the variance of a linear fit within eight phase bins. We remove all systems with weak periodicities (see Devor 2005 for details), and with one exception (T-Lyr114413), all the systems whose optimal period is found to be longer than half their LC duration. In this way, we are able to filter out many of the non-periodic variables.

The AoV algorithm is most effective in identifying the periods of LCs with long duration features, such as semi-detached EBs and pulsating stars. The BLS algorithm, in contrast, is effective at identifying periodic systems whose features span only a brief portion of the period, such as detached EBs and transiting planets (see Figure 1). However, the BLS algorithm is easily fooled by outlier data points, identifying them as short duration features. For this reason, the BLS algorithm has a significantly higher rate of false positives than AoV, especially for long periods, which have only a few cycles over the duration of the observations.
Therefore, we limit the search range of the BLS algorithm to periods shorter than 12 days, although as Figure 1 illustrates, its efficiency at locating EBs rapidly declines at periods greater than 10 days.

In step 2, we address the ambiguity between EBs with identical components in a circular orbit, and EBs with extremely disparate components. The phased LC of EBs with identical components contains two identical eclipses, whereas the phased LC of EBs with disparate components will have a secondary eclipse below the photometric noise level. These two cases are degenerate, since doubling the period of a disparate system will result in an LC that looks like an equal-component system. In the pipeline, we handle this problem by doubling such entries; one with the period found in step (1), and another with twice that period. Both of these entries proceed through the pipeline independently. In many cases, after additional processing by the following steps, one of these entries will emerge as being far less likely than the other (see Appendix A), at which point it is removed. But in cases where photometry alone cannot determine which is correct, one needs to perform spectroscopic follow up to break the ambiguity. In particular, a double-lined spectrum would support the equal-component hypothesis.

Step 3 is performed using DEBiL, which fits the fractional radii $\left(r_{1,2}\right)$ and observed magnitudes $\left(\operatorname{mag}_{1,2}\right)$ of the EB's stellar components, their orbital inclination $(i)$ and eccentricity $(e)$, and their epoch $\left(t_{0}\right)$ and argument of periastron $(\omega)$. DEBiL first produces an initial guess for these parameters, and then iteratively improves the fit using the downhill simplex method (Nelder \& Mead 1965) with simulated annealing (Kirkpatrick et al. 1983; Press et al. 1992).

In step 4, we fine-tune the period $(P)$ using a method based on eclipse timing ${ }^{7}$, which we describe below. In order to produce an accurate EB model in step 9, it is necessary to know the system's period with greater accuracy than that produced in step 1 . If we neglect to fine-tune the period, the eclipses may be out of phase with respect to one another, and so the phased eclipses will appear broadened. Our timing method employs the DEBiL model produced in step 3, and uses it to find the difference between the observed and calculated $(O-C)$ eclipse epochs. This is done by minimizing the chi-squared fit of the model to the data points in each eclipse, while varying only the model's epoch of periastron. When the period estimate is off by a small quantity $(\Delta P)$, the $O-C$ difference increases by $\Delta P$ each period. This change in the $O-C$ over time can be measured from the slope of the linear regression, which is expected to equal $\Delta P / P$. Thus measuring such an $O-C$ slope will yield the desired period correction (see Figure 2).

If the EB has an eccentric orbit, the primary and secondary eclipse will separate on the $O-C$ plot, and form two parallel lines with a vertical offset of $\Delta t$ (see Figure 3). We measure this offset and use it as a sensitive method to detect orbital eccentricities. In particular, the value of $\Delta t$ constrains $e \cos \omega$, which in turn provides a lower limit for the system's eccentricity (Tsesevich 1973):

$$
e \cos \omega \simeq \frac{\pi}{2} \frac{\Delta t}{P}
$$

This formula assumes an orbital inclination of $i=90^{\circ}$, making it a good approximation for eclipsing binaries. We use this method, in combination with DEBiL, to identify the

\footnotetext{
7 The source code and running examples are available online at http://www.cfa.harvard.edu/ jdevor/Timing.html.
} 


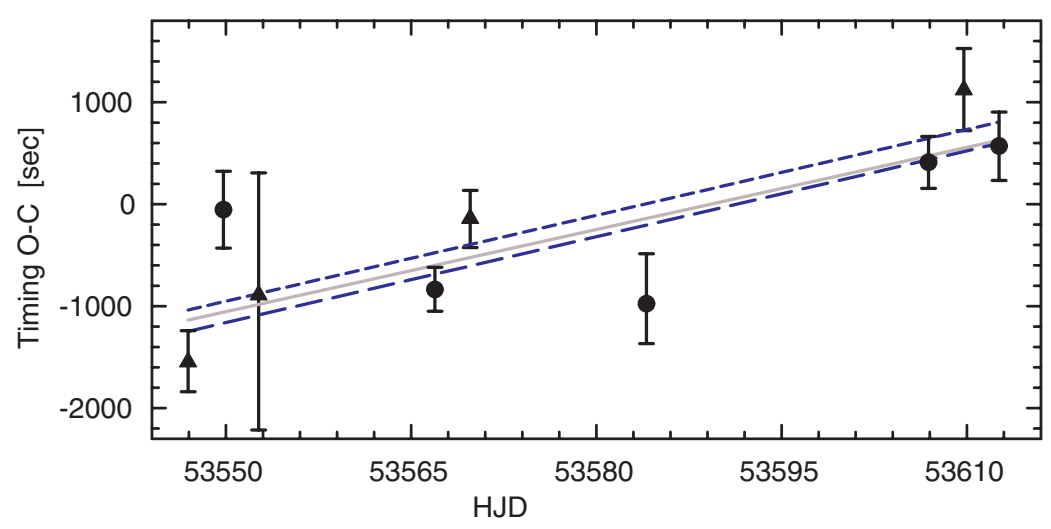

Figure 2. An eclipse timing plot produced in step 4, showing the $O-C$ residuals of the primary eclipses (circles) and the secondary eclipses (triangles). Here, T-Lyr1-14962 is shown with an assumed period of 5.710660 days, as measured with an AoV periodogram. The slope of the residuals indicates that the assumed period is inaccurate. The gray solid line is predicted by the best circular-orbit model, whereas the dashed lines are predicted by the best eccentric-orbit model (compare to Figure 3). After correction, we get a fine-tuned period of 5.712516 days. This $0.03 \%$ correction is small but significant in that without having had this correction, the eclipses would have smeared out and widened.

(A color version of this figure is available in the online journal)

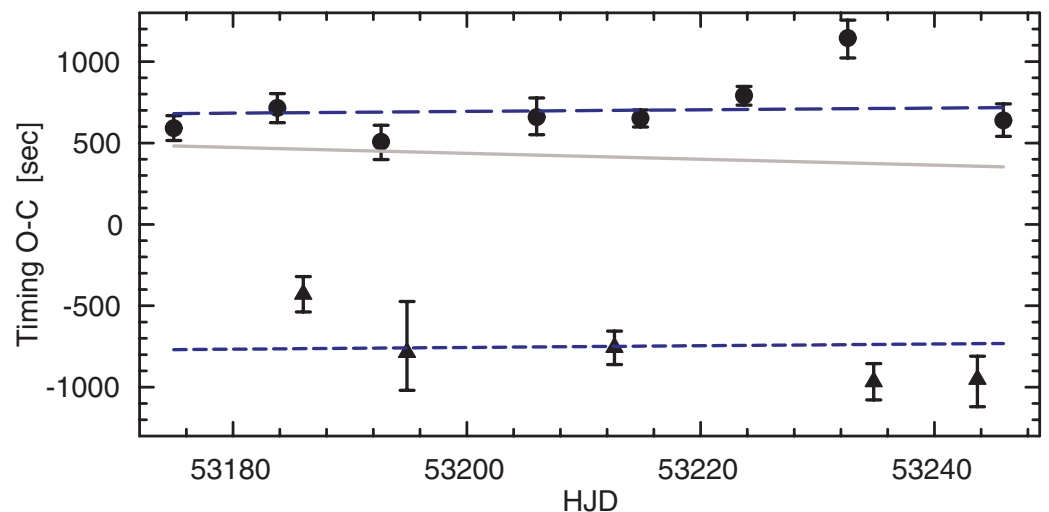

Figure 3. An eclipse timing plot for T-Cyg1-01373, with an assumed period of 4.436013 days. In contrast to Figure 2, the slope here is consistent with zero, thus indicating that the period does not need to be fine-tuned. However, the $O-C$ offset between the primary (circles) and secondary (triangle) eclipses is significant $(1449 \mathrm{~s})$, indicating that this EB has an eccentric orbit. The reduced chi-squared of the best circular-orbit model (gray solid line) is $\chi_{v}^{2}=12.9$, while the reduced chi-squared of the best eccentric-orbit model (dashed lines) is $\chi_{v}^{2}=0.95$. Applying the $O-C$ timing offset to Equation (1) provides a lower limit to the binary's orbital eccentricity: $e \geqslant|e \cos \omega| \simeq 0.00594$.

(A color version of this figure is available in the online journal)

eccentric EBs in the catalog (see Table 3). However, in cases where the eclipse timing measures $|e \cos \omega|<0.005$, or when the eccentricity is consistent with zero, we assume that the EB is non-eccentric, and model it using a circular orbit. We further discuss the physics of these systems in Section 3.2.

Step 5 is identical to step 3, except that it uses the revised period from step 4 . This step provides an improved fit to the LCs, as evidenced by an improved chi-squared value in over $70 \%$ of the cases.

In step 6, we locate and remove non-EB sources that seem to be periodic due to photometric contamination by true EBs. Such contaminations result from overlapping point-spread functions (PSF) that cause each source to partially blend into the other. These cases can be easily identified with a program that scans through pairs of targets ${ }^{8}$, and selects those that both have similar periods (see description below) and are separated by an angle that is smaller than twice the PSF. We found 14 such pairs, all

\footnotetext{
8 We ran a brute force scan, which required $\mathrm{O}\left(N^{2}\right)$ iterations. But by employing a data structure that can restrict the scan to nearby pairs, it is possible to perform this scan in only $\mathrm{O}(N)$ iterations, assuming that such pairs are rare.
}

of which were separated by less than $41^{\prime \prime}$, which is well within twice the TrES PSF $\left(60^{\prime \prime}\right)$, while the remaining pairs with similar periods were separated by over $450^{\prime \prime}$. Upon inspection, all 14 of the pairs we found had similar eclipse shapes, indicating that we had no false positives. Between each pair, we identify the LC with shallower eclipses (in magnitudes) as being contaminated and remove it from the catalog.

We define periods as being similar if the difference between them is smaller than their combined uncertainty. We estimate the period uncertainty using the relation: $\varepsilon_{P} \propto P^{2} / T$, where $T$ is the time interval between the initial and the final observations. One arrives at this relation by noticing that when phasing the LC, the effect of any perturbation from the true period will grow linearly with the number of periods in the LC (see step 4). This amplified effect will become evident once it reaches some fraction of the period itself, in other words, when $\varepsilon_{P}(T / P) \propto P$. A typical TrES LC with a revised period will have a proportionality constant of approximately $1 / 1000$. In order to avoid missing contaminated pairs (false negatives), we adopt in this step, the extremely liberal proportionality constant of unity. 
Table 3

Eccentric EBs

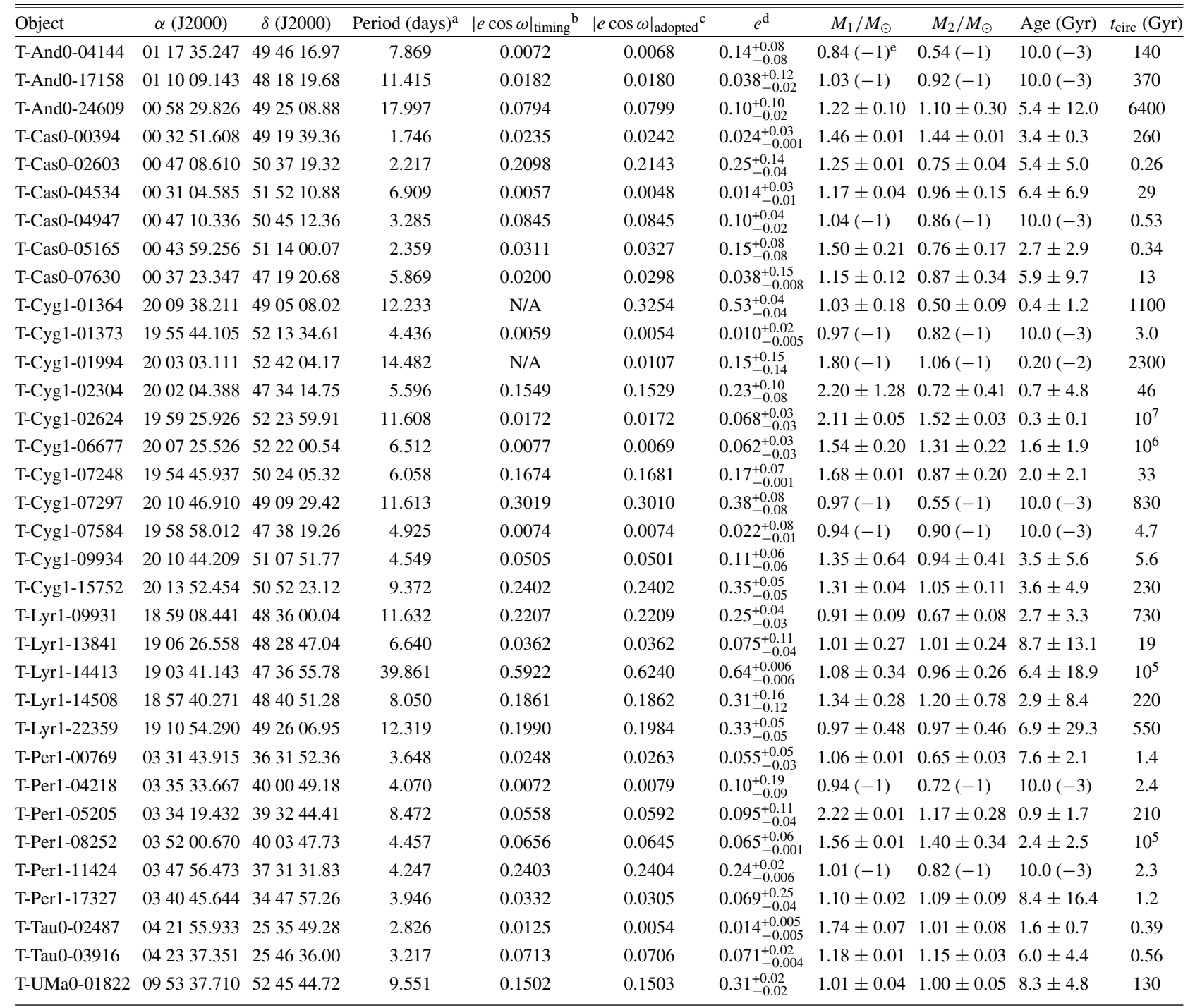

Notes.

a The full precision of the measured period is listed in the online journal (see Table 7), together with its uncertainty and the epoch of the center of eclipse (see Appendix B).

${ }^{b}$ Measurements made using the eclipse timing of step 4. Although these values are approximations, they do not suffer from nearly as much numerical error as the DEBiL measurement, and are therefore usually accurate. "N/A" marks LCs for which there were too few eclipses to be able to apply the timing method.

c The adopted value is a combination of the values measured with the timing method and with DEBiL.

$\mathrm{d}$ The uncertainties of the eccentricities are non-Gaussian, since they have a strict lower bound $(e \geqslant|e \cos \omega|)$. We truncated the quoted lower uncertainties at this value, though even at this truncated value the real uncertainty is beyond $1 \sigma$.

e When the most likely model is at the edge of the parameter space, MECI is not able to bound the solution, and therefore cannot estimate the uncertainties.

We mark $(-3)$ when the upper limit was reached, $(-2)$ when the lower limit was reached, and $(-1)$ if one of the other parameters is at its limit.

In step 7, we conduct a visual inspection of all the LC fits. Most EBs were successfully modeled and were included into the catalog as is. About $1 \%$ of the LCs analyzed had misidentified periods, as a result of failures of the period-finding method of step 1. In most of these cases, the period finder indicated either a harmonic of the true period or a rational multiple of a solar or sidereal day. In such cases, we use an interactive periodogram ${ }^{9}$ to find the correct period and then reprocess the LCs through the pipeline. Some entries were

\footnotetext{
9 LC, created by Grzegorz Pojmanski.
}

misidentified at step 2 as being ambiguous, even though they have a detectable secondary eclipse or have slightly unequal eclipses. In these cases, the erroneous doubled entry was removed. Lastly, some of the EBs were not fit sufficiently well with DEBiL in step 5. These cases were typically due to clustered outlier data points, systematic noise, or severe activity of a stellar component (e.g., flares or spots), which caused DEBiL to produce erroneous initial model parameters. These cases were typically handled by having DEBiL produce the initial model parameters from a more smoothed version of the LC. 
In step 8, we match each system, through its coordinates, with the corresponding source in the Two Micron All Sky Survey catalog (2MASS; Skrutskie et al. 2006). This was done to obtain both accurate target positions and observational magnitudes. These magnitude measurements are then used to derive the colors of each EB, which are incorporated into the MECI analysis, as well as to estimate the EB's distance modulus (step 9). To this end, 2 MASS provides a unique combination of high astrometric accuracy $\left(\sim 0.1^{\prime \prime}\right)$ together with high photometric accuracy $(\sim 0.015 \mathrm{mag})$ at multiple near-infrared bands, all while maintaining a decent photometric resolving power $\left(\sim 3^{\prime \prime}\right)$. By employing these near-infrared bands, we both inherently reduce the detrimental effects of stellar reddening, and are able to correct for much of the remaining extinction by fitting for the Galactic interstellar absorption.

In order to use the measurements from the 2MASS custom $J$, $H$, and $K_{s}$ filters, we converted them to the equivalent ESO-filter values so that they could be compared to the isochrone table values used in the MECI analysis. This conversion was done using approximate linear transformations (Carpenter 2001). However, the colors of three EBs (T-And0-10336, T-Cyg102304, and T-Per1-05205) were so anomalous that they did not permit a reasonable model solution; thus, we chose not to include any color information in their MECI analyses.

In addition to its brightness, we also look up each EB's proper motion. Although proper motion is not required for any of the pipeline analyses, it provides a useful verification for low-mass candidates (see Section 3.1). These systems are expected to have large proper motions, since they must be nearby to be observable in this magnitude-limited survey. The most extreme such case in the catalog is CM Draconis (T-Dra0-01363), which has a proper motion of over

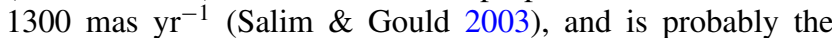
lowest mass system in our catalog. To this end, we match each system to the Second U.S. Naval Observatory CCD Astrograph Catalog (UCAC release 2.4; Zacharias et al. 2004). When there is no match with UCAC, we use the more comprehensive but less accurate U.S. Naval Observatory photographic sky survey (USNO-B release 1.0; Monet et al. 2003). These matches are made using the more accurate aforementioned adopted 2MASS coordinates. However, because of their increased observational depth, and the fact that some high-proper motion targets are expected to have moved multiple arcseconds in the intervening decades, we chose to match each target to the brightest ( $R$-band) source within 7.5". It should be noted that the position of CM Draconis shifted by more than $22^{\prime \prime}$ and had to be matched manually, though $90 \%$ of the matches were separated by less than $0.6^{\prime \prime}$, and $98 \%$ were separated by less than $2^{\prime \prime}$ (see Figure 4).

The proper motions garnered from these databases can be combined with distance estimates $(D)$, to calculate the absolute transverse velocity $\left(v_{\mathrm{tr}}\right)$ of a given EB:

$$
v_{\text {tr }} \simeq 4.741 \mathrm{~km} \mathrm{~s}^{-1}\left(\frac{\mathrm{PM}}{1 \mathrm{mas} \mathrm{yr}^{-1}}\right)\left(\frac{D}{1 \mathrm{kpc}}\right),
$$

where PM is the system's angular proper motion. In the catalog, we list the right ascension and declination components $\left(\mathrm{PM}_{\alpha}\right.$ and $\mathrm{PM}_{\delta}$, respectively), so as to allow one to compute the system's direction of motion in the sky. The value of PM can be computed from its components, using $\mathrm{PM}^{2}=\mathrm{PM}_{\delta}^{2}+\mathrm{PM}_{\alpha}^{2} \cos ^{2} \delta$, where $\delta$ is the system's declination. When applying this formula, one should be aware that USNO-B folds the $\cos \delta$ coefficient into its listed $\mathrm{PM}_{\alpha}$, while UCAC does not.

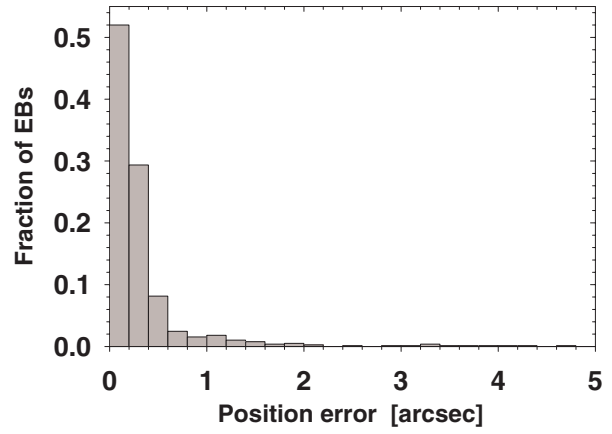

Figure 4. The distribution of the catalog position errors when matching targets to the proper motion databases. In some cases, the position errors are dominated by the motion of the EB during the intervening years.

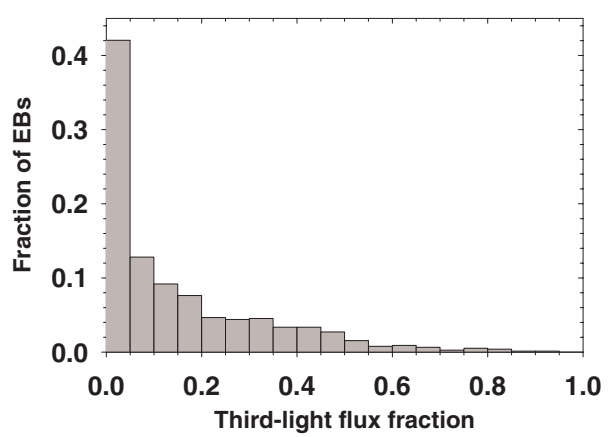

Figure 5. The distribution of the $R$-band third-light flux fraction in the catalog LCs. This fraction was calculated by summing the fluxes of all the USNO-B sources within $30^{\prime \prime}$ of the target, excluding the target, and dividing this value by the total flux within $30^{\prime \prime}$, including the target. The resulting fraction ranges from 0 to 1 .

Finally, we incorporate the USNO-B photometric $B$ - and $R$-magnitude measurements into our catalog to provide a rough estimate of the optical brightness of each target. USNO-B lists two independent measurements in each of these filter; however, in some cases one or both of these measurements failed. When both measurements are available, we average them for improved accuracy. However, each measurement has a large photometric uncertainty of $\sim 0.3 \mathrm{mag}$; thus, even these averaged values will have errors that are over an order of magnitude larger than the photometric measurements of 2MASS. For this reason, and because of the increased effect of stellar reddening, we chose not to incorporate these data into the MECI analysis. However, USNO-B's high photometric resolution $\left(\sim 1^{\prime \prime}\right)$ enabled us to detect many sources that blended with our targets in the TrES exposures. By summing the $R$-band fluxes of all the USNO-B sources within $30^{\prime \prime}$ of each target, we estimated the fraction of third-light included in each LC (see Figure 5). Note that this measure provides only a lower bound to the true thirdlight fraction, as some EBs are expected to have additional close hierarchical components that would not be resolved by USNO-B. For most of the catalog targets, the third-light flux fraction was found to be small $(<10 \%)$. We therefore conclude that stellar blending will usually have only a minor effect on the MECI analysis results; however, users should be aware of the potential biases in the calculated properties of highly blended targets. Though it was not applied to this catalog, in principle, given a third-light flux fraction at a well-determined LC phase, one could correct for the effects of blending.

In step 9, we analyze the LCs with MECI. We refer the reader to the full description of this method in Devor \& Charbonneau 

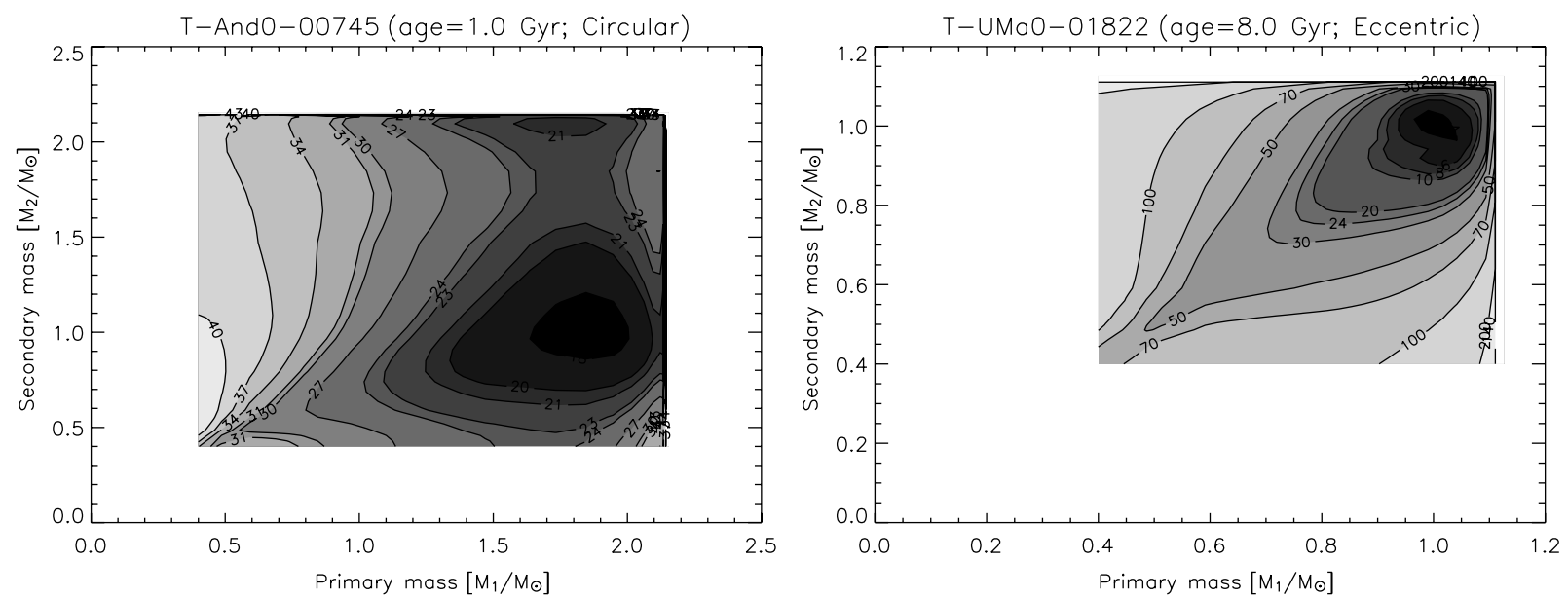

Figure 6. MECI likelihood contour plots of a typical circular-orbit EB (T-And0-00745; left) and eccentric-orbit EB (T-UMa0-01822; right). There is no significant difference in the way MECI handles these cases, and both usually have a single contour minimum. The plots shown here have the ages set to the values that produced the lowest MECI minima.
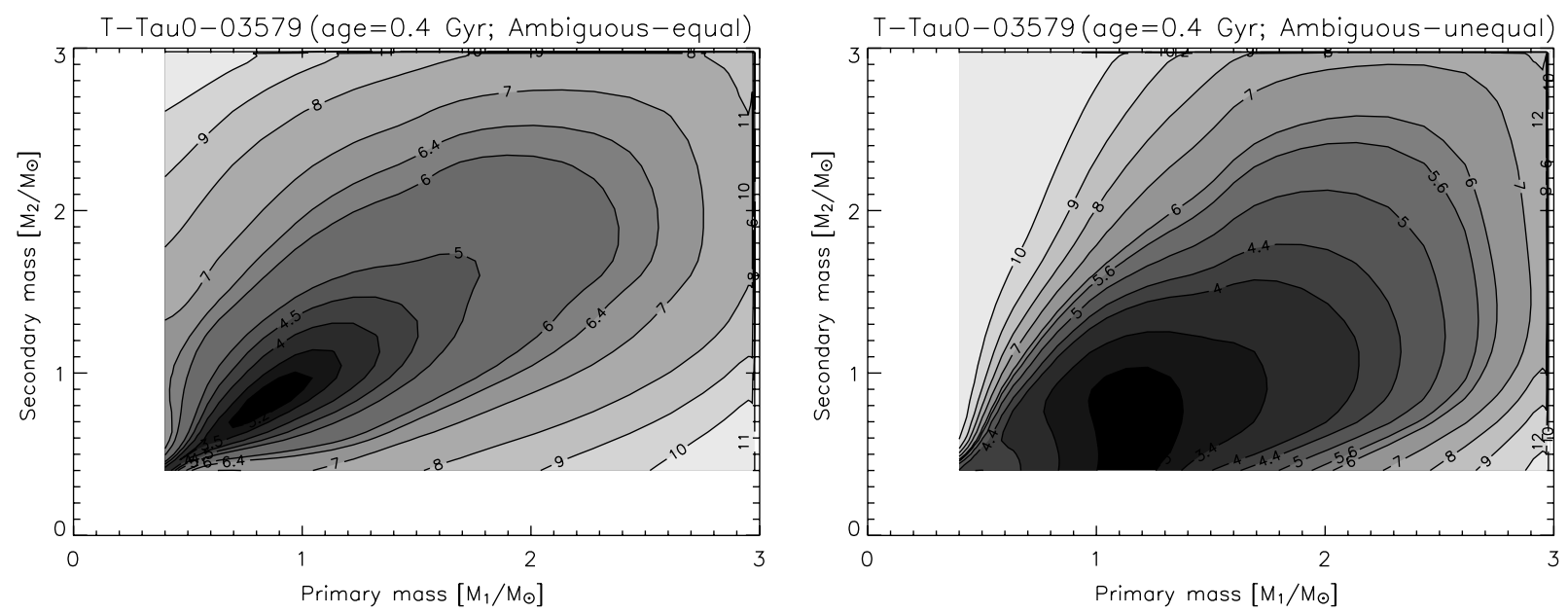

Figure 7. MECI likelihood contour plots of a typical ambiguous EB (T-Tau0-03579). These plots show the effect of assuming that the binary components are equal (left) or unequal (right). Note that the equal-component solution will have a nearly symmetric contour around the diagonal, while the unequal-component solution can provide only an upper limit to the secondary component's mass, in this case $M_{2} \lesssim 1 M_{\odot}$. The plots shown here have the ages set to the values that produced the lowest MECI minima.

(2006a, 2006b), and provide here only a brief outline. Given an observed EB LC and out-of-eclipse colors, MECI will iterate through a range of values for the EB age and the masses of its two components. By looking up their radii and luminosities in theoretical isochrone tables, MECI simulates the expected LC and combined colors, and selects the model that best matches the observations, as measured by the chi-squared statistic. Or, more concisely, MECI searches the $\left(M_{1}, M_{2}\right.$,age)-parameter space for the chi-squared global minimum of each EB. Figures 6 and 7 show constant-age slices through such a parameter space. Once found, the curvature of the global minimum along the parameter space axes is used to determine the uncertainties of the corresponding parameters.

The MECI analysis makes two important assumptions. The first is that EB stellar components are coeval, which has been shown to generally hold for close binaries (Claret \& Willems 2002). When this assumption is violated, MECI will often not be able to find an EB model that successfully reproduces the LC eclipses. Such systems, which may be of interest in their own right, make up $\sim 3 \%$ of the catalog and are further discussed later in this section. The second assumption is that there is no significant reddening, or third-light blended into the observations (i.e. from a photometric binary or hierarchical triple). Such blending in the LC will make the eclipses shallower, which produces an effect very similar to that of the EB having a grazing orbit. Thus, it will cause the measured orbital inclination to be erroneous, although it should rarely otherwise affect the results of the MECI analysis significantly. However, the MECI analysis is sensitive to color biases caused by stellar reddening and blending.

We reduce both these biases by incorporating 2MASS colors (see step 8), which are both less suspectable to reddening than optical colors, and suffers from significantly less blending than TrES, as the radius of the 2MASS photometric aperture is $\sim 20$ times smaller than that of TrES. We then attempt to further mitigate this problem by analyzing each EB twice, using different relative LC/color information weighting values (see Devor \& Charbonneau 2006b for further details). We first run MECI with the default weighting value $(w=10)$, and then run MECI again with an increased LC weighting $(w=100)$ thereby 
decreasing the relative color weighting. Finally, we adopt the solution that has a smaller reduced chi-square. Typically, the results of the two MECI analyses are very similar, indicating that the observed colors are consistent with those predicted by the theoretical isochrones. In such cases, the color information provides an important constraint, which significantly reduces the parameter uncertainties. However, when there is a significant color bias, the default model will not fit the observed data as well as the model that uses a reduced weighting of the color information. In such a case, the reduced color information model, which has a smaller chi-squared, is adopted. Following this procedure, we find that in $\sim 9 \%$ of our EBs, the reduced color information model provides a better fit, indicating that while significant color-bias is uncommon, it is a source of error that should not be ignored.

By default, we had MECI use the Yonsei-Yale (Yi et al. 2001; Kim et al. 2002) isochrone tables of solar metallicity stars. Although they successfully describe stars in a wide range of masses, these tables become increasingly inaccurate for lowmass stars, as the stars become increasingly convective. For this reason, we re-analyze EBs for which both components were found to have masses below $0.75 M_{\odot}$, using instead the Baraffe et al. (1998) isochrone tables, assuming a convective mixing length equal to the pressure scale height. Our EB models also take into account the effects of the limb darkening of each of the stellar components. To this end, we employ the ATLAS (Kurucz 1992) and PHOENIX (Claret 1998, 2000) tables of quadratic limb-darkening coefficients.

As previously mentioned, once we know the absolute properties of an EB system, we are able to estimate its distance (Stebbing 1910; Paczynski 1997), and thus such systems can be considered standard candles. We use the extinction coefficients of Cox (2000), assuming the standard Galactic ISM optical parameter, $R_{V}=3.1$, to create the following system:

$$
\begin{gathered}
\operatorname{mag}_{J}-\operatorname{Mag}_{J}=\Delta \mathrm{Mag}+0.282 \cdot A(V) \\
\operatorname{mag}_{H}-\operatorname{Mag}_{H}=\Delta \mathrm{Mag}+0.176 \cdot A(V) \\
\operatorname{mag}_{K}-\operatorname{Mag}_{K}=\Delta \mathrm{Mag}+0.108 \cdot A(V)
\end{gathered}
$$

where $\Delta \mathrm{Mag}$ is the extinction-corrected distance modulus, and $A(V)$ is the $V$-mag absorption due to Galactic interstellar extinction. The estimated distance can then be solved using $D=10 p c \cdot 10^{\Delta \mathrm{Mag} / 5}$. Because we have three equations for only two unknowns, we adopt the solution that minimizes the sum of the squares of the residuals. In some cases, we remove one of the bands as being an outlier (i.e. if it would have resulted in a negative absorption), after which we are still able to solve the systems. But in cases where we need to remove two bands, we set $A(V)=0$ in order to solve for the distance modulus. Although this method has a typical uncertainty of $10 \%$ to $20 \%$, it can be applied to EBs that are far more distant and dim than are accessible in other methods, such as parallax measurement. It can be used to map broad features of the Galaxy, and identify binaries that are in the Galactic halo. This method can also be applied to a clustered group of EBs, whereby averaging their distances will reduce the distance uncertainty to the cluster as the inverse square root of the number of EBs measured.

In step 10, we perform a final quality check for the EB model fits, and classify them into seven groups.

I. Eccentric: EBs with unequally-spaced eclipses.

II. Circular: EBs with equally-spaced but distinct eclipses.

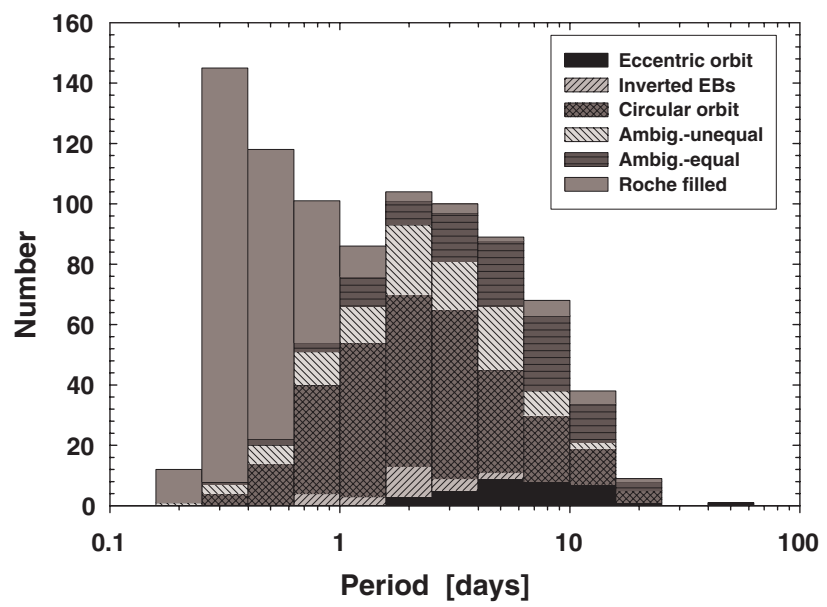

Figure 8. The EB orbital period distribution within the catalog. Each bin is subdivided to show the number of binaries belonging to each of the classification groups described in Section 2. Note that the ambiguous-equal and ambiguousunequal entries represent the same stars, with entries in the former group having double the period of the latter. Note also how the Roche-lobe-filling EBs dominate the sub-day bins, and have a long tail stretching well above 10 day periods. Furthermore, the circular-orbit EBs have a period distribution peak of at $\sim 2$ days, while the eccentric orbit EBs peak at $\sim 5$ days. This is likely due to the orbital circularization that occurs preferentially in short-period systems (see also Figures 22 and 23).

III. Ambiguous-unequal: EBs with undetected secondary eclipses.

IV. Ambiguous-equal: EB with equally-spaced and indistinguishable eclipses.

V. Inverted: detached EBs that are not successfully modeled by MECI.

VI. Roche-lobe-filling: non-detached EBs that are filling at least one Roche-lobe.

VII. Abnormal: EBs with atypical out-of-eclipse distortions.

We list the model parameters for the EBs of groups I-IV in the electronic version of this catalog (see full description in Appendix B). The EBs of groups V-VII could not be well modeled by MECI; therefore, we list only their coordinates and periods, so that they can be followed up.

Figure 8 illustrates the period distribution of these seven groups. Note however that both the orbital geometry of EBs (eclipse probability $\propto P^{-2 / 3}$ ), and the limited duration of the TrES survey data ( $\leqslant 90$ days; varies from field to field; see Table 1), act to suppress the detection of binaries with longer periods. An added complication for single-telescope surveys is that about half of the EBs with periods close to an integer number of days will not be detectable, as they eclipse only during the daytime. This EB distribution is consistent with the far deeper OGLE II field catalog (Devor 2005), where the long tail of Roche-lobe-filling systems has recently been explained by Derekas et al. (2007) as being the result of a strong selection toward detecting eclipsing giant stars.

Group I contains the eccentric EBs identified in step (4) as having centers of eclipse that are separated by a duration significantly different from half an orbital period (see Figures 9-11). This criterion is sufficient for demonstrating eccentricity, but not necessary, since we miss systems for which $\cos \omega \simeq 0$ (see Equation (1)). Fortunately, we are able to detect eccentricities in well-detached EBs with $|e \cos \omega| \geqslant 0.005$, using eclipse timing. Therefore, assuming that $\omega$ is uniformly distributed, we are approximately $67 \%$ complete for $e=0.01$, and over $92 \%$ 

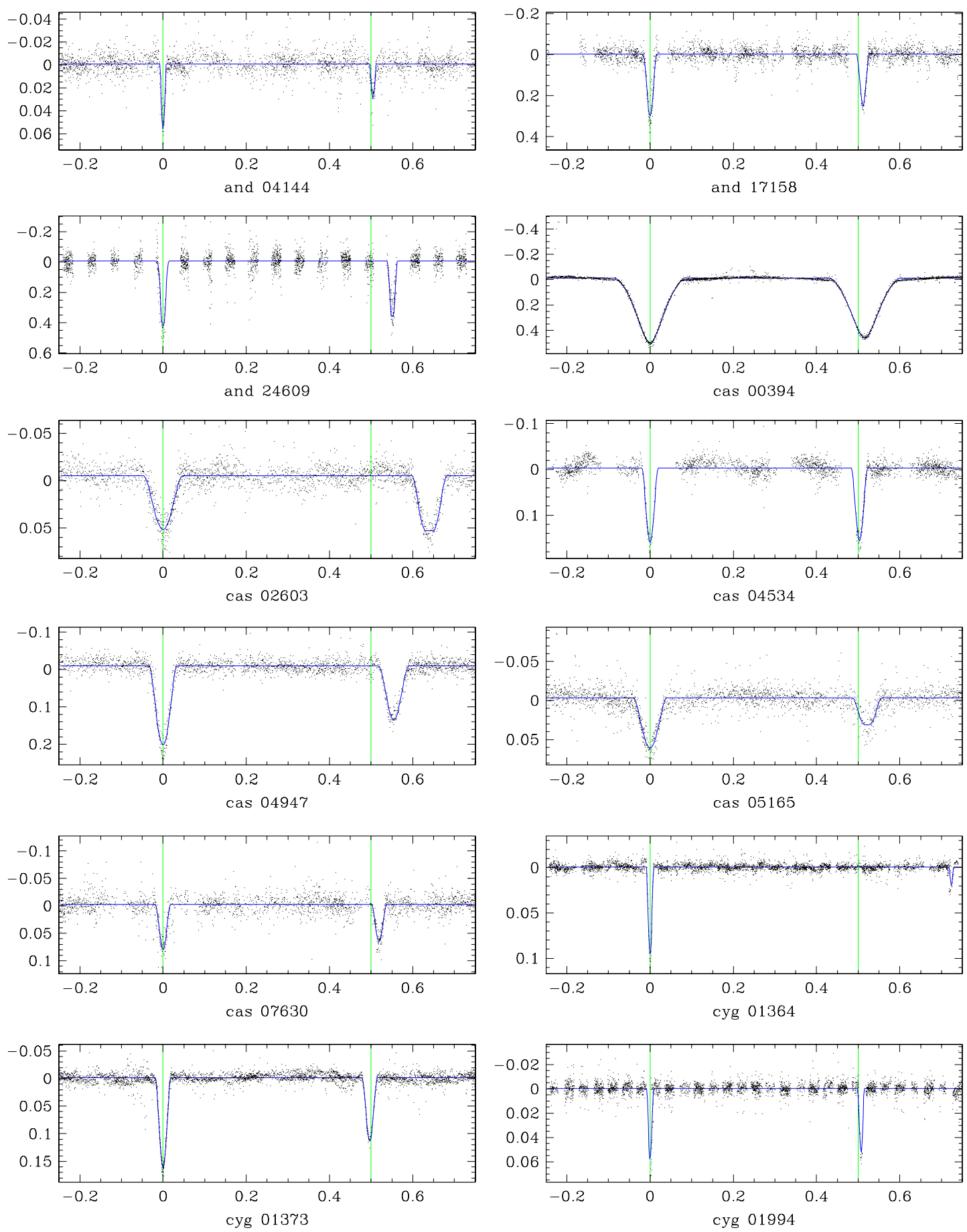

Figure 9. Eccentric EBs (panel 1). Note how the secondary eclipse is not at phase 0.5, as it would be in circular orbit EBs.

(A color version of this figure is available in the online journal)

complete for $e=0.04$. In principle, it would be possible to be $100 \%$ complete for these systems by measuring the differences in their eclipse durations; however, this measurement is known to be unreliable (Etzel 1991) and so would likely contaminate this group with false positives. Group II consists of all such circular-orbit EBs that were successfully fit by a single MECI model (see Figure 12).

EBs with only one detectable eclipse can potentially be modeled in two alternative ways. One way is to assume very unequal stellar components, which have a very shallow undetected secondary eclipse (group III). Since we cannot estimate the eccentricity of such systems, we assume that they have circular orbits. The other way is to assume that the period at hand is twice the correct value, and that the components are nearly equal (group IV). The entries of such ambiguous LCs were doubled in step 2, so that these two solutions would be independently processed through the pipeline (see Figure 13). Therefore, these two groups have a one-to-one correspondence 



Figure 10. Eccentric EBs (panel 2).

(A color version of this figure is available in the online journal)

between them, although only one entry of each pair can be correct. Resolving this ambiguity may not always be possible without spectroscopic data. In some cases, we were able to resolve this ambiguity using either a morphological or a physical approach. The morphological approach consists of manually examining the LCs of group IV for any asymmetries in the two eclipses (e.g., width, depth, or shape), or in the two plateaux between the eclipses (e.g., perturbations due to tidal effects, reflections, or the "O'Connell effect"). The physical approach consists of applying our understanding of stellar evolution in order to exclude entries that cannot be explained through any coeval star pairing (see Appendix A). Either way, once one of the two models has been eliminated, the other model is moved into group II and is adopted as a non-ambiguous solution. It 

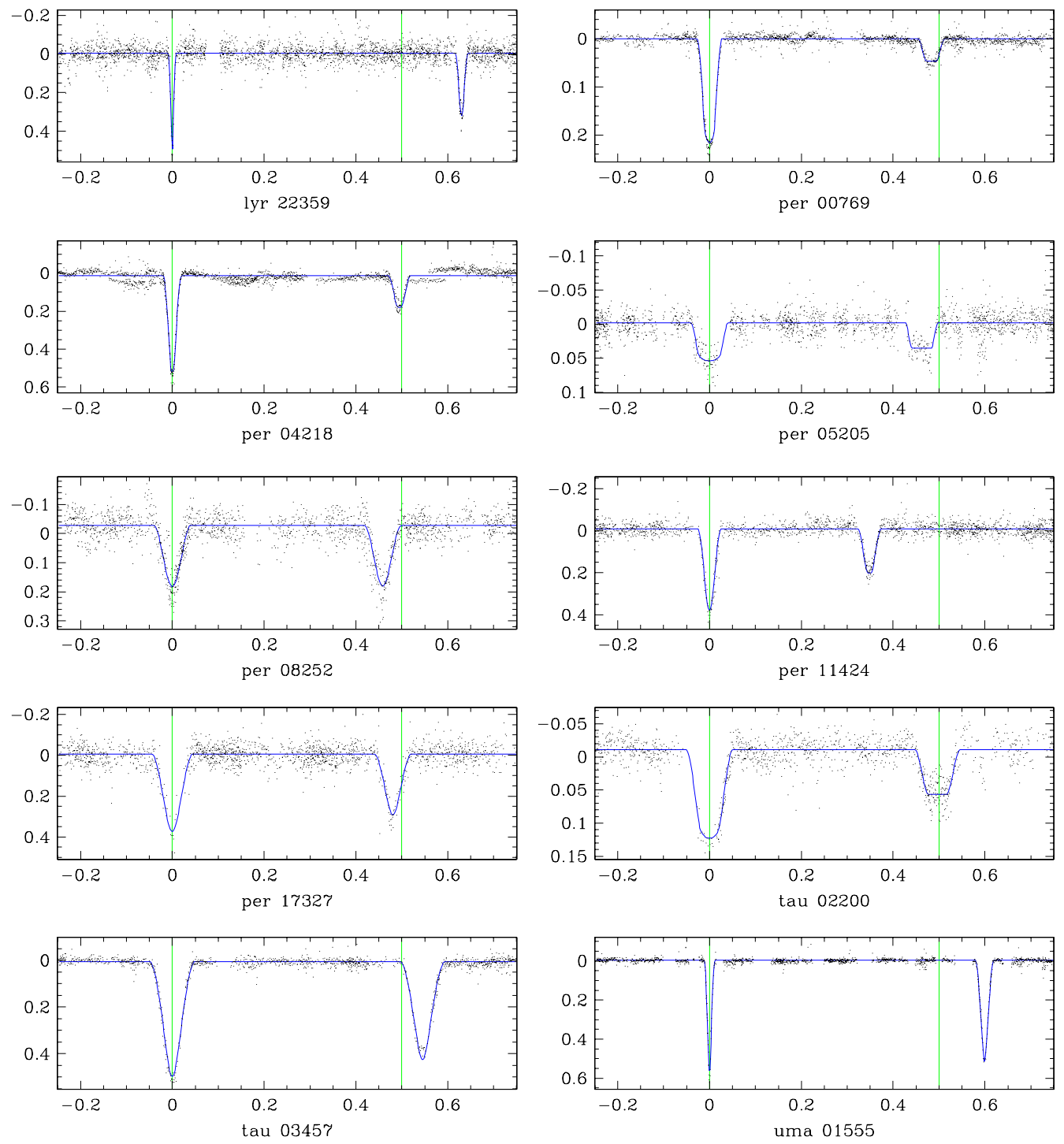

Figure 11. Eccentric EBs (panel 3).

(A color version of this figure is available in the online journal)

is interesting to note that when analyzing the two models with MECI, the equal-component solution (group IV) has masses approximately equal to the primary component of the unequalcomponent solution (group III). The mass of the unequalcomponent solution's secondary component will typically be the smallest value listed in the isochrone table, as this configuration will produce the least detectable secondary eclipse.

Group V consists of detached EBs that cannot be modeled by two coeval stellar components. As mentioned earlier, we can reject the single-eclipse solution for EBs with sufficiently deep eclipses (see Appendix A). This argument can be further extended to cases where we can detect both eclipses in the LC, but where one is far shallower than the other. In some cases, no two coeval main-sequence components will reproduce such an LC, but unlike the previous case, since both eclipses are seen, we cannot conclude that the period needs to be doubled. Such systems are likely to have had mass transfer from a sub-giant component onto a main-sequence component through Roche-lobe overflow, to the point where currently the main-sequence component has become significantly more massive and brighter than it was originally (Crawford 1955). This process will cause the components to effectively behave as non-coeval stars, even though they have in fact the same chronological age. In extreme cases, the originally lower-mass mainsequence component can become more massive than the subgiant, and thus swap their original primary/secondary designations, so that the main-sequence component is now the primary component. We call such systems "inverted" EBs, and place them into group V (see Figure 14). This phenomenon is often referred to in the literature as the "Algol paradox," though we choose not to adopt this term so as to avoid confusing it with the term "Algol-type EB" (EA), which is defined by the General Catalogue of Variable Stars (GCVS; Kukarkin \& Parengo 1948; Samus 2006) as being the class of all well-detached EBs.

Group VI contains the EBs that have at least one component filling its Roche-lobe (see Figure 15). Such system cannot be well fit by either DEBiL or MECI since they assume that the binary components are detached, and so neglect tidal and 

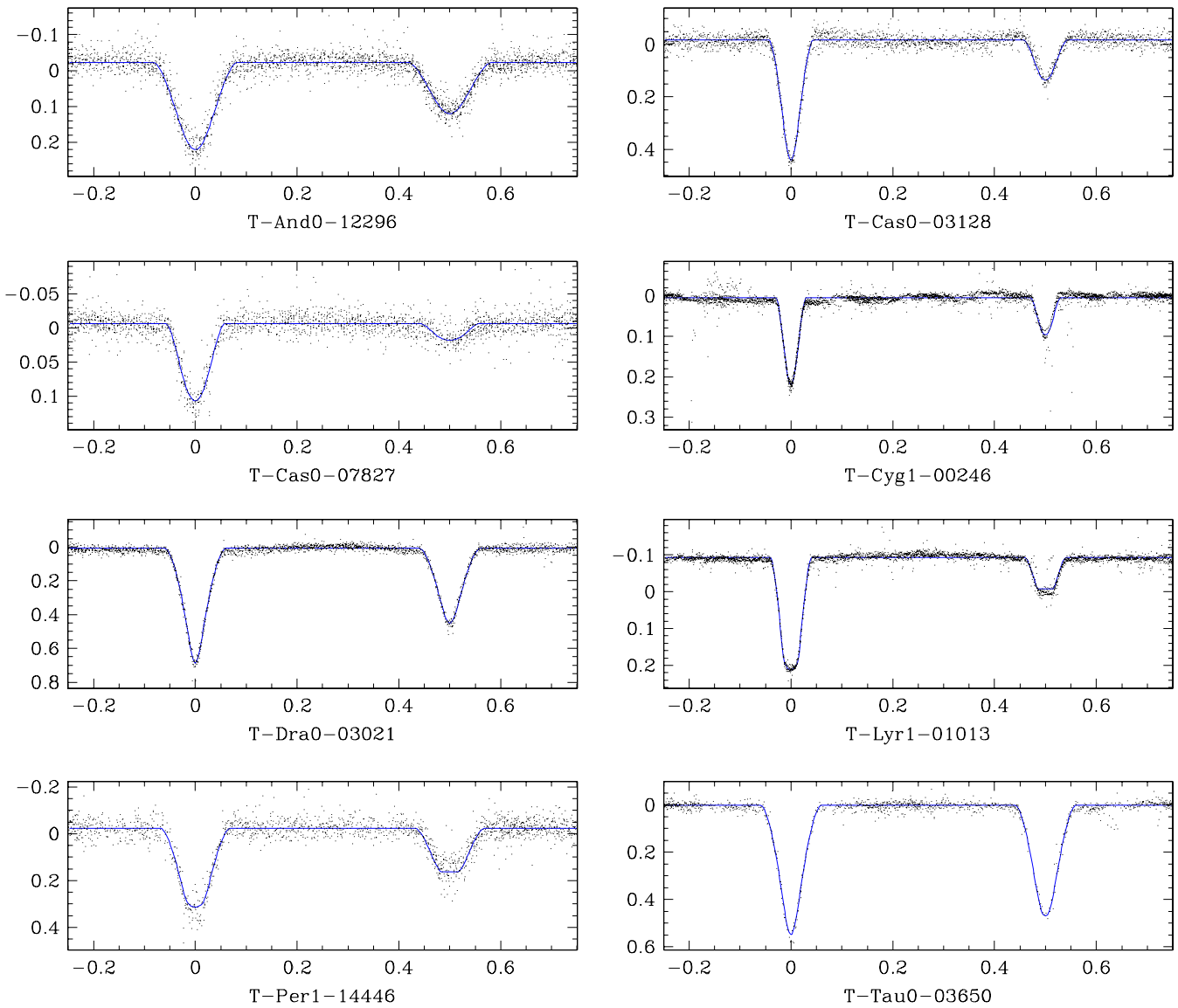

Figure 12. Examples of unambiguous EBs with circular orbits, with their best-fit MECI models (solid line).

(A color version of this figure is available in the online journal)

rotational distortions, gravity darkening, and reflection effects. These systems must be separated from the rest of the catalog since their resulting best-fit models will be poor and therefore their evaluated physical attributes will likely be erroneous. In a similar fashion to Tamuz et al. (2006), we detect these systems automatically by applying the Eggleton (1983) approximation for the Roche-lobe radius, and place in group VI all the systems for which at least one of the EB components has filled its Rochelobe (see Figure 16), that is, if either one of the following two inequalities occurs:

$$
r_{1}>\frac{0.49 q^{-2 / 3}}{0.6 q^{-2 / 3}+\ln \left(1+q^{-1 / 3}\right)}
$$

or

$$
r_{2}>\frac{0.49 q^{2 / 3}}{0.6 q^{2 / 3}+\ln \left(1+q^{1 / 3}\right)},
$$

where $q=M_{2} / M_{1}$ is the EB components' mass ratio. Since we expect non-detached EBs to be biased toward evolving, highermass stellar components, we estimated $q$ using the early-type mass-radius power-law relation found in binaries (Gorda \& Svechnikov 1998): $q \simeq\left(r_{2} / r_{1}\right)^{1.534}$. Although, in principle, we could have estimated $q$ directly from the EB component masses resulting from the MECI analysis, we chose not to, since as stated above, the analysis of such systems is inaccurate. The analytic approximation we used, though crude, proved to be remarkably robust, as we found only five false negatives and no false positives when visually inspecting the LCs. We found many more false positives/negatives when using the alarm criteria suggested by Devor (2005) or Mazeh et al. (2006), both of which attempt to identify bad model fits by evaluating spatial correlations of the model's residuals.

Finally, group VII contains systems visually identified as EBs (i.e. having LCs with periodic flux dips), yet having atypical LC perturbations that indicate the existence of additional physical phenomena (see Figures 17 and 18). For lack of a better descriptor, we call such systems "abnormal" (see further information in Section 3.3). This group is different from the previous six in that we cannot automate their classification, and their selection is thus inherently subjective. In 15 of the 20 systems, we were able to approximately model the LCs, and included them in one of the aforementioned groups. In these cases, users should be aware that these model may be biased by the phenomenon that brought about their LC distortion.

\section{RESULTS}

We identified and classified a total of $773 \mathrm{EBs}^{10}$. These systems consisted of 734 EBs with circular orbits, 34 detached EBs with eccentric orbits (group I; Table 3), and 5 unclassified abnormal EBs (group VII; Table 5). We marked 15 of the detached EBs with circular orbits as also being abnormal. Of the 734 EBs with circular orbits, we classify 290 as unambiguous

\footnotetext{
10 The observed LCs, fitted models, and model residuals of each of these EBs are shown at http://www.cfa.harvard.edu/ jdevor/Catalog.html.
} 

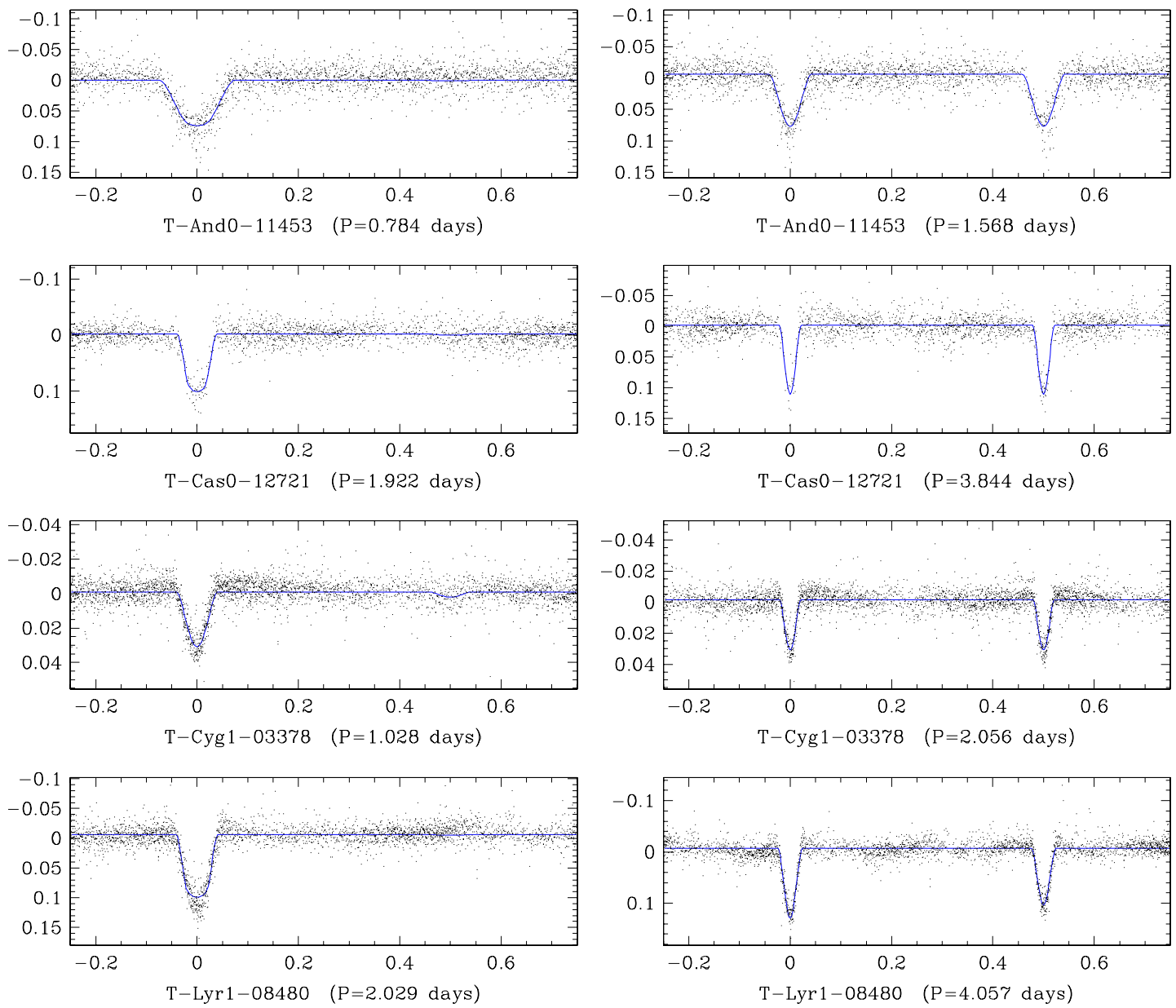

Figure 13. Examples of ambiguous EBs. Left column: assuming very unequal components. Right column: assuming approximately equal components with double the period.

(A color version of this figure is available in the online journal)
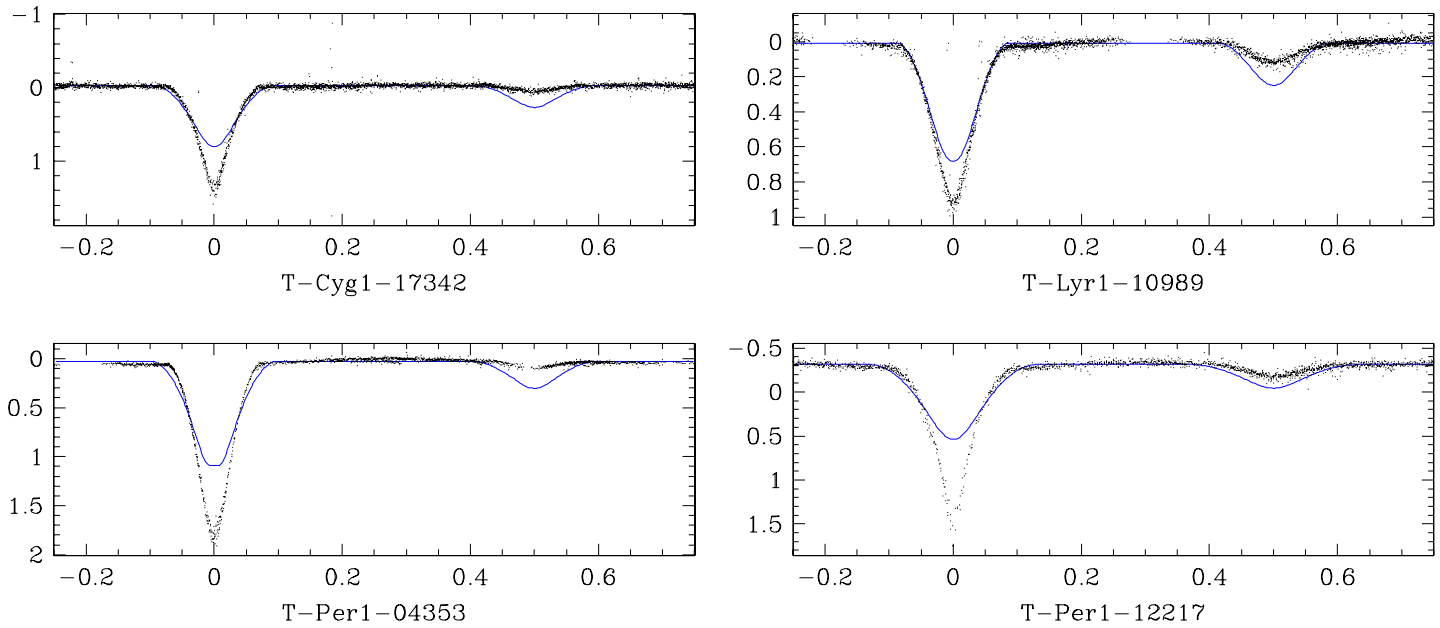

Figure 14. Examples of EBs classified as inverted EBs. We included the unsuccessful best-fit MECI model (solid curve) as an approximate reference to illustrate the LC of a corresponding binary that has had no mass transfer. Note how the model LC is unable to achieve a sufficiently deep primary eclipse, while producing a secondary eclipse that is too deep.

(A color version of this figure is available in the online journal)

detached EBs (group II; Table 7), 103 as ambiguous detached $\mathrm{EBs}$, for which we could not determine photometrically if they consisted of equal or disparate components (groups III and IV; Table 6), 23 as inverted EBs (group V; Table 8), and 

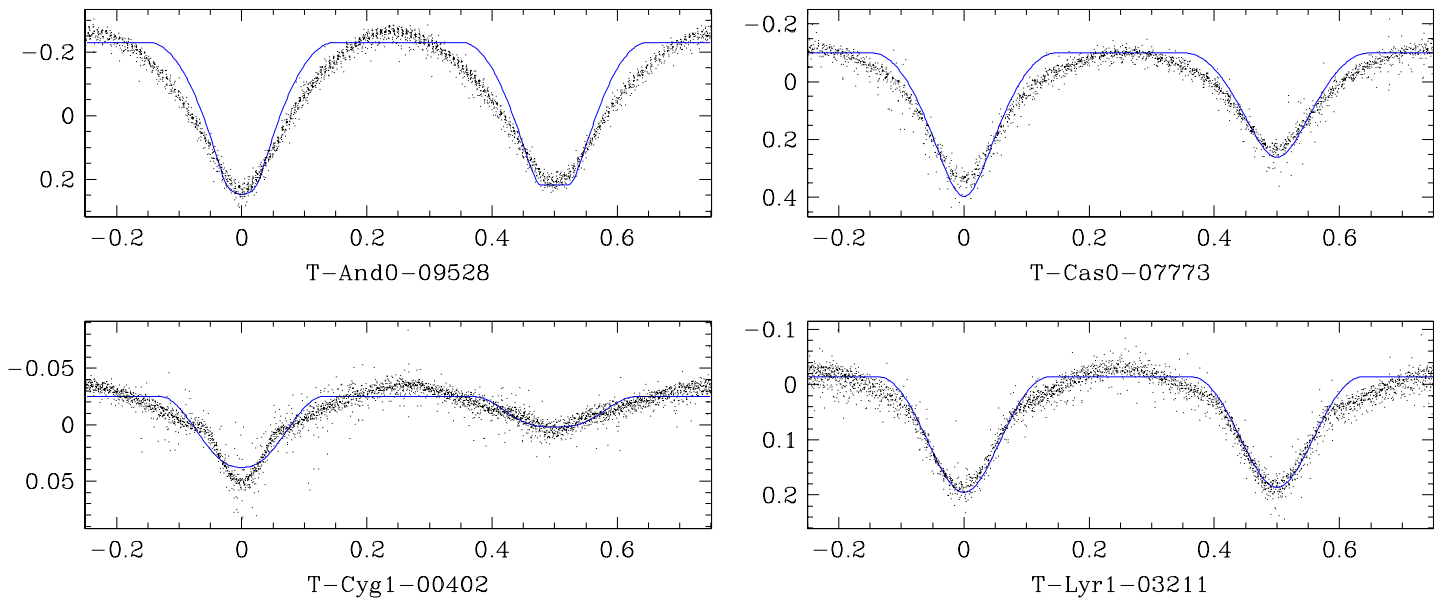

Figure 15. Examples of EBs that are assumed to have filled at least one of their Roche-lobes. We included, for illustration purposes only, their best-fit MECI models (solid line). These models were not adopted since they neglect tidal distortions, reflections, and gravity-darkening effects, and so produce a poor fit to the data. (A color version of this figure is available in the online journal)

Table 4

EBs that Fill at Least One of Their Roche-Lobes (First 20)

\begin{tabular}{lccc}
\hline \hline Object & $\alpha(\mathrm{J} 2000)$ & $\delta(\mathrm{J} 2000)$ & Period (days) \\
\hline T-And0-03774 & 005901.029 & 464717.08 & 1.362 \\
T-And0-04813 & 011637.880 & 473323.43 & 0.552 \\
T-And0-05140 & 010322.258 & 445624.31 & 0.981 \\
T-And0-05153 & 011848.278 & 493936.86 & 0.492 \\
T-And0-05343 & 005255.122 & 480137.68 & 0.824 \\
T-And0-07638 & 010927.871 & 492033.81 & 0.403 \\
T-And0-07892 & 005615.567 & 483910.73 & 0.380 \\
T-And0-08330 & 011915.949 & 480017.45 & 0.630 \\
T-And0-08652 & 005658.855 & 490505.00 & 0.335 \\
T-And0-09528 & 012209.328 & 471429.86 & 0.918 \\
T-And0-10071 & 011450.412 & 491746.28 & 0.387 \\
T-And0-10206 & 005555.724 & 494946.56 & 0.859 \\
T-And0-10511 & 011916.430 & 470746.27 & 0.563 \\
T-And0-10722 & 010403.859 & 483713.04 & 1.062 \\
T-And0-11354 & 011805.168 & 461014.66 & 0.331 \\
T-And0-11476 & 010732.106 & 455544.93 & 6.380 \\
T-And0-11599 & 010928.113 & 461824.85 & 0.280 \\
T-And0-11617 & 010728.020 & 452240.35 & 0.503 \\
T-And0-12453 & 011712.316 & 464235.43 & 0.448 \\
T-And0-12769 & 005258.164 & 444411.26 & 0.325 \\
\end{tabular}

Notes. The complete EB dataset is available in the online journal with Table 7. This table shows a portion of the EBs that fill at least one of their Roche-lobes subsample.

318 as non-detached (group VI; Table 4). With the exception of the abnormal EBs, which were selected by eye, we use an automated method to classify each of these groups (see Section 2 for details). Our mass estimates for the primary and secondary components are plotted in Figure 19.

The EB discovery yield (the fraction of LCs found to be EBs) varies greatly from field to field, ranging from $0.72 \%$ for Cygnus, to $0.15 \%$ for Corona Borealis (see Table 2). This variation is strongly correlated with Galactic latitude, where fields near the Galactic plane have larger discovery yields than those that are farther from it (see Figure 20). This effect is likely due to the fact that fields closer to the Galactic plane contain a higher fraction of early-type stars. These early-type stars are both physically larger, making them more likely to be eclipsed, and are more luminous, which causes them to

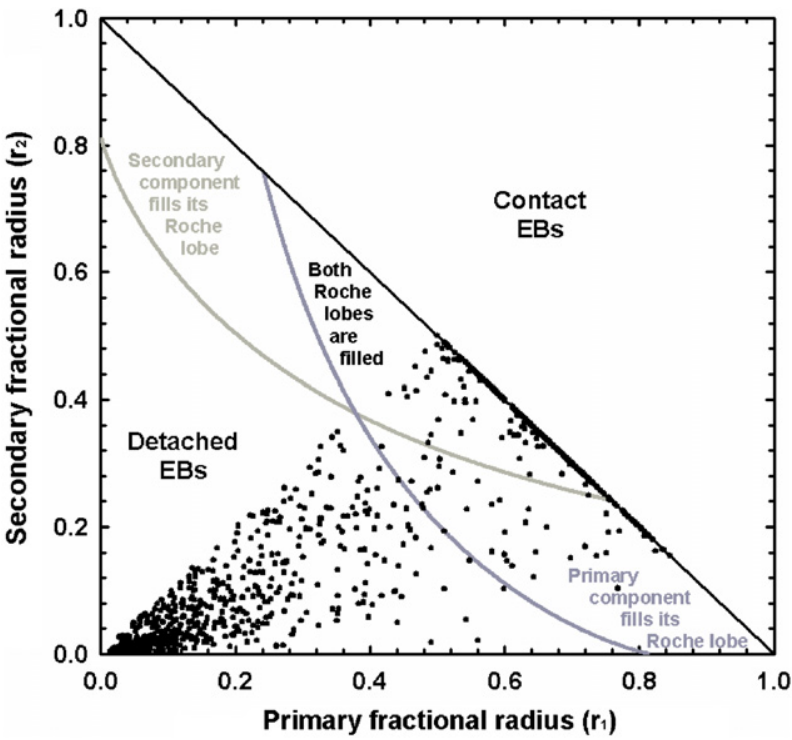

Figure 16. The criterion applied in Equations (6) and (7) to determine whether one or both the EB components have filled their Roche-lobe, and thus need to be placed into group (6).

(A color version of this figure is available in the online journal)

produce brighter and less noisy LCs, thereby enabling the detection of EBs with shallower eclipses. Furthermore, much of the residual scatter can be attributed to the variation in the observed duration of each field (see Table 1). That is, we find additional EBs, with longer periods, in fields that were observed for a longer duration.

Currently, 88 of the cataloged EBs (11\%) appear in either the International Variable Star Index ${ }^{11}$ (VSX), or in the SIMBAD ${ }^{12}$ astronomical database (Table 9). However, only 49 systems $(6 \%)$ have been identified as being variable. Not surprisingly, with few exceptions, these targets were among the brightest sources of the catalog. Using only photometry, it is often notoriously difficult to distinguish non-detached EBs from

\footnotetext{
11 Maintained by the American Association of Variable Star Observers (AAVSO).

12 Maintained by the Centre de Données astronomiques de Strasbourg (CDS).
} 
Table 5

Abnormal EBs

\begin{tabular}{|c|c|c|c|c|c|c|}
\hline Object & $\alpha(\mathrm{J} 2000)$ & $\delta(\mathrm{J} 2000)$ & $\begin{array}{l}\text { Period } \\
\text { (days) }\end{array}$ & $\begin{array}{c}\text { Classified } \\
\text { in Catalog? }\end{array}$ & $\begin{array}{c}\text { In SIMBAD/VSX? } \\
\quad(\text { See Table 9) }\end{array}$ & Notes \\
\hline T-And0-00920 & 011730.677 & 470331.61 & 24.073 & No & No & Large asymmetric reflection ( $0.1 \mathrm{mag})$ offset eclipse \\
\hline T-And0-04594 & 011610.713 & 485218.97 & 3.910 & Yes & No & Spots/active \\
\hline T-And0-11476 & 010732.106 & 455544.93 & 6.380 & Yes & No & Tilted plateaux (spots?) \\
\hline T-Cas0-13944 & 002948.990 & 504954.06 & 1.739 & Yes & No & Irregular eclipse depths \\
\hline T-Cyg1-07584 & 195858.012 & 473819.26 & 4.925 & Yes & No & Large persistent spot \\
\hline T-Cyg1-08866 & 200836.448 & 492935.79 & 2.876 & Yes & No & Offset eclipse ${ }^{\mathrm{a}}$ \\
\hline T-Dra0-03105 & 162302.558 & 592723.44 & 0.485 & No & Yes & Unequal eclipses ${ }^{\mathrm{b}} /$ semi-detached \\
\hline T-Dra0-04520 & 164957.960 & 562645.56 & 3.113 & Yes & No & Tilted plateaux (spots?) \\
\hline T-Her0-03497 & 165228.391 & 445129.63 & 7.853 & Yes & No & Unequal plateaux $^{\mathrm{c}}$ \\
\hline T-Her0-08091 & 165152.608 & 470147.98 & 2.694 & Yes & No & Offset eclipse \\
\hline T-Lyr1-00359 & 191533.695 & 443701.30 & 1.062 & Yes & Yes & Large recurring spots $(\sim 0.05 \mathrm{mag})$ \\
\hline T-Lyr1-02800 & 190818.809 & 471248.16 & 4.876 & No & No & Semi-detached/unequal plateaux (spots?) \\
\hline T-Lyr1-05984 & 185350.481 & 453320.90 & 1.470 & No & No & Unequal eclipses $\mathrm{b} /$ semi-detached \\
\hline T-Lyr1-13166 & 190228.120 & 465857.75 & 0.310 & No & No & Unequal plateaux; misshapen eclipse (persistent spot?) \\
\hline T-Lyr1-15595 & 190605.267 & 490408.95 & 9.477 & Yes & No & Offset eclipse \\
\hline T-Per1-00750 & 034745.543 & 350037.08 & 1.929 & Yes & Yes & Spots/active \\
\hline T-Per1-08789 & 035433.282 & 390741.53 & 2.645 & Yes & No & Tilted plateaux \\
\hline T-UMa0-03090 & 100852.180 & 524552.49 & 0.538 & Yes & Yes & Unequal plateaux \\
\hline
\end{tabular}

Notes.

The complete EB dataset is available in the online journal with Table 7. This table shows a portion of the abnormal EBs subsample.

${ }^{a}$ Even when the LC plateaux are not flat, due to tidal distortion or reflections, the system's mirror symmetry normally guarantees that the eclipses will occur during a plateau minimum or maximum. When, as in these cases, the eclipses are significantly offset from the plateau minima/maxima we can conclude that some mechanism, perhaps severe tidal lag, is breaking the system's symmetry.

${ }^{b}$ Might not be an EB. This LC could be due to non-sinusoidal pulsations.

c The two LC plateaux between the eclipses, have a significantly different mean magnitude. This may be due to one or both components being tidally locked, and having a persistent spot or surface temperature variation at specific longitudes.

Table 6

Ambiguous EBs (First 10)

\begin{tabular}{|c|c|c|c|c|c|c|c|}
\hline Version & Object & $\alpha(\mathrm{J} 2000)$ & $\delta(\mathrm{J} 2000)$ & Period (days) & $M_{1} / M_{\odot}$ & $M_{2} / M_{\odot}$ & Age (Gyr) \\
\hline A & T-And0-00657 & 010606.159 & 473159.37 & 6.725 & $2.50(-1)^{\mathrm{a}}$ & $0.74(-1)$ & $0.20(-2)$ \\
\hline B & T-And0-00657 & 010606.159 & 473159.37 & 13.456 & $1.92(-1)$ & $1.92(-1)$ & $0.20(-2)$ \\
\hline A & T-And0-01203 & 010334.745 & 483239.27 & 3.505 & $1.86 \pm 0.09$ & $0.56 \pm 0.10$ & $0.89 \pm 0.83$ \\
\hline B & T-And0-01203 & 010334.745 & 483239.27 & 7.011 & $1.90 \pm 0.12$ & $0.66 \pm 0.19$ & $0.80 \pm 1.13$ \\
\hline A & T-And0-06017 & 011248.217 & 495807.16 & 2.543 & $1.40 \pm 0.35$ & $0.52 \pm 0.77$ & $3.49 \pm 4.28$ \\
\hline B & T-And0-06017 & 011248.217 & 495807.16 & 5.085 & $1.18 \pm 0.71$ & $1.12 \pm 0.85$ & $3.12 \pm 11.15$ \\
\hline A & T-And0-06500 & 012556.083 & 492331.74 & 5.337 & $0.97 \pm 0.20$ & $0.49 \pm 0.53$ & $7.71 \pm 16.33$ \\
\hline B & T-And0-06500 & 012556.083 & 492331.74 & 10.674 & $1.01 \pm 0.30$ & $0.93 \pm 0.45$ & $0.74 \pm 1.60$ \\
\hline A & T-And0-06680 & 005548.153 & 450248.57 & 4.551 & $1.16 \pm 0.04$ & $0.51 \pm 0.20$ & $6.09 \pm 8.91$ \\
\hline B & T-And0-06680 & 005548.153 & 450248.57 & 9.104 & $1.16 \pm 0.09$ & $0.96 \pm 0.29$ & $6.24 \pm 10.78$ \\
\hline A & T-And0-08053 & 011359.402 & 455143.43 & 4.116 & $1.14(-1)$ & $0.40(-2)$ & $6.00(-1)$ \\
\hline B & T-And0-08053 & 011359.402 & 455143.43 & 8.231 & $1.09 \pm 0.55$ & $1.05 \pm 0.64$ & $3.22 \pm 16.37$ \\
\hline A & T-And0-08417 & 010139.041 & 450332.98 & 2.053 & $1.01(-1)$ & $0.47(-1)$ & $10.00(-3)$ \\
\hline B & T-And0-08417 & 010139.041 & 450332.98 & 4.106 & $1.01(-1)$ & $0.90(-1)$ & $10.00(-3)$ \\
\hline A & T-And0-09365 & 010100.459 & 451424.77 & 1.887 & $1.05 \pm 0.03$ & $0.43 \pm 0.39$ & $8.74 \pm 16.06$ \\
\hline B & T-And0-09365 & 010100.459 & 451424.77 & 3.774 & $1.05 \pm 0.05$ & $0.93 \pm 0.52$ & $9.47 \pm 23.55$ \\
\hline A & T-And0-10518 & 010744.417 & 484458.11 & 0.194 & $0.90(-1)$ & $0.40(-2)$ & $0.40(-1)$ \\
\hline B & T-And0-10518 & 010744.417 & 484458.11 & 0.387 & $0.45 \pm 0.27$ & $0.45 \pm 0.28$ & $0.27 \pm 0.54$ \\
\hline A & T-And0-11453 & 010542.744 & 445402.26 & 0.784 & $1.12(-1)$ & $0.40(-2)$ & $7.00(-1)$ \\
\hline B & T-And0-11453 & 010542.744 & 445402.26 & 1.568 & $1.02 \pm 0.43$ & $1.01 \pm 0.32$ & $8.81 \pm 14.54$ \\
\hline
\end{tabular}

Notes. The complete EB dataset is available in the online journal with Table 7. This table shows a portion of the ambiguous EBs subsample. A: unequal eclipse model, assuming an unseen secondary eclipse. B: equal eclipse model, with double the period of the unequal model. a When the most likely model is at the edge of the parameter space, MECI is not able to bound the solution, and therefore cannot estimate the uncertainties. We mark $(-3)$ when the upper limit was reached, $(-2)$ when the lower limit was reached, and $(-1)$ if one of the other parameter is at its limit.

pulsating variables that vary sinusoidally in time, such as type-C RR Lyrae. Furthermore, unevenly spotted stars may also cause false positive identifications, especially in surveys with shorter durations. Ultimately, spectroscopic follow-up 
Table 7

Circular EBs (First 20)

\begin{tabular}{|c|c|c|c|c|c|c|c|c|c|}
\hline Object & $\alpha(\mathrm{J} 2000)$ & $\delta(\mathrm{J} 2000)$ & Period (days) & $M_{1} / M_{\odot}$ & $M_{2} / M_{\odot}$ & Age (Gyr) & $\begin{array}{l}\text { Proper Motion } \\
\text { Source Catalog }\end{array}$ & $\begin{array}{c}\mathrm{PM}_{\alpha} \\
\left(\text { mas yr }^{-1}\right)\end{array}$ & $\begin{array}{c}\mathrm{PM}_{\delta} \\
\left(\mathrm{mas} \mathrm{yr}^{-1}\right)\end{array}$ \\
\hline T-And0-00194 & 012012.816 & 483641.36 & 2.145 & $2.07 \pm 0.02$ & $0.97 \pm 0.02$ & $0.59 \pm 0.12$ & UCAC & 28.4 & -12.2 \\
\hline T-And0-00459 & 011124.845 & 465749.44 & 3.655 & $1.20 \pm 0.01$ & $1.19 \pm 0.01$ & $5.35 \pm 1.13$ & UCAC & -1.6 & -20.6 \\
\hline T-And0-00745 & 010345.076 & 445041.14 & 2.851 & $1.86 \pm 0.23$ & $1.02 \pm 0.22$ & $1.04 \pm 0.72$ & UCAC & -6.6 & -4.8 \\
\hline T-And0-01461 & 010615.353 & 450825.66 & 5.613 & $1.47 \pm 0.01$ & $1.45 \pm 0.08$ & $2.76 \pm 2.72$ & UCAC & -11.4 & 2.8 \\
\hline T-And0-01554 & 011704.999 & 455406.20 & 1.316 & $0.90(-1)^{\mathrm{a}}$ & $0.84(-1)$ & $10.00(-3)$ & UCAC & -44.6 & -40.8 \\
\hline T-And0-01597 & 011032.071 & 464953.18 & 3.503 & $1.55 \pm 0.03$ & $1.54 \pm 0.01$ & $2.37 \pm 0.76$ & UCAC & 2.9 & -5.5 \\
\hline T-And0-02699 & 010644.813 & 473108.61 & 1.759 & $1.18 \pm 0.02$ & $0.53 \pm 0.07$ & $5.21 \pm 3.37$ & UCAC & 0.2 & -6.8 \\
\hline T-And0-02798 & 012118.345 & 484805.63 & 2.860 & $1.04 \pm 0.10$ & $0.65 \pm 0.13$ & $6.14 \pm 9.51$ & UCAC & 6.3 & -8.1 \\
\hline T-And0-03526 & 012017.451 & 473923.32 & 1.536 & $1.04 \pm 0.02$ & $0.84 \pm 0.02$ & $6.29 \pm 2.37$ & UCAC & 17.9 & -11.1 \\
\hline T-And0-04046 & 005520.157 & 474453.20 & 3.916 & $1.30 \pm 0.09$ & $1.25 \pm 0.12$ & $3.10 \pm 4.31$ & UCAC & -3.8 & -7.3 \\
\hline T-And0-04594 & 011610.713 & 485218.97 & 3.910 & $1.05(-1)$ & $0.82(-1)$ & $10.00(-3)$ & UCAC & 1.5 & -1.9 \\
\hline T-And0-04829 & 011515.228 & 474558.97 & 0.678 & $0.99(-1)$ & $0.92(-1)$ & $10.00(-3)$ & UCAC & -23.8 & 44.4 \\
\hline T-And0-05241 & 005634.679 & 463702.91 & 1.454 & $1.56 \pm 0.01$ & $1.47 \pm 0.31$ & $2.69 \pm 7.01$ & UCAC & -4.5 & -0.5 \\
\hline T-And0-05794 & 011211.763 & 473230.94 & 1.053 & $2.06 \pm 0.19$ & $1.08 \pm 0.54$ & $1.08 \pm 1.92$ & UCAC & -0.4 & -1.4 \\
\hline T-And0-06039 & 012337.548 & 482537.73 & 4.923 & $1.22 \pm 0.05$ & $1.08 \pm 0.31$ & $5.33 \pm 7.17$ & UCAC & -2.5 & -5.0 \\
\hline T-And0-06340 & 010155.269 & 491838.23 & 5.437 & $1.33(-1)$ & $0.40(-2)$ & $4.00(-1)$ & UCAC & 0.3 & -2.9 \\
\hline T-And0-06538 & 012058.907 & 492908.89 & 18.669 & $1.33 \pm 0.15$ & $0.97 \pm 0.17$ & $3.38 \pm 3.45$ & UCAC & 1.1 & -6.8 \\
\hline T-And0-06632 & 012236.840 & 475253.29 & 1.669 & $1.69 \pm 0.01$ & $1.45 \pm 0.24$ & $2.21 \pm 1.02$ & UCAC & -7.2 & -7.6 \\
\hline
\end{tabular}

Notes. ${ }^{a}$ When the most likely model is at the edge of the parameter space, MECI is not able to bound the solution, and therefore cannot estimate the uncertainties. We mark $(-3)$ when the upper limit was reached, $(-2)$ when the lower limit was reached, and $(-1)$ if one of the other parameter is at its limit.

(This table is available in its entirety in a machine-readable form in the online journal. A portion is shown here for guidance regarding its form and content)

Table 8

Inverted EBs

\begin{tabular}{lccc}
\hline \hline Object & $\alpha(\mathrm{J} 2000)$ & $\delta(\mathrm{J} 2000)$ & Period (days) \\
\hline T-And0-13653 & 005957.881 & 450341.53 & 3.342 \\
T-Cas0-02069 & 004917.959 & 503902.92 & 2.830 \\
T-Cas0-03012 & 004541.832 & 510135.40 & 1.108 \\
T-Cas0-04618 & 004622.661 & 503917.57 & 2.798 \\
T-Cas0-07780 & 003418.779 & 520035.72 & 1.852 \\
T-Cas0-19045 & 002144.707 & 503229.55 & 0.785 \\
T-Cas0-19668 & 004801.342 & 470611.58 & 1.848 \\
T-Cas0-21651 & 002634.895 & 463842.69 & 1.155 \\
T-Cyg1-01956 & 195329.106 & 474849.86 & 2.045 \\
T-Cyg1-02929 & 201157.009 & 480703.59 & 4.263 \\
T-Cyg1-17342 & 194954.197 & 505328.08 & 2.220 \\
T-Her0-05469 & 165451.245 & 432035.89 & 0.899 \\
T-Lyr1-04431 & 191216.047 & 494223.58 & 0.903 \\
T-Lyr1-05887 & 185210.489 & 474816.67 & 1.802 \\
T-Lyr1-07179 & 184914.039 & 452438.61 & 1.323 \\
T-Lyr1-10989 & 190622.791 & 454153.82 & 2.015 \\
T-Lyr1-11067 & 185253.489 & 475126.58 & 2.241 \\
T-Per1-04353 & 034504.887 & 374715.91 & 2.953 \\
T-Per1-06993 & 034059.668 & 391235.90 & 2.125 \\
T-Per1-09366 & 034920.305 & 395541.97 & 2.374 \\
T-Per1-12217 & 032859.454 & 373742.14 & 1.690 \\
T-Tau0-00686 & 040713.870 & 291832.44 & 5.361 \\
T-UMa0-00127 & 093806.716 & 560107.32 & 0.687 \\
\hline
\end{tabular}

Notes. The complete EB dataset is available in the online journal with Table 7. This table shows a portion of the inverted EBs subsample.

will always be necessary to confirm the identification of such variables.

We highlight three groups of EBs as potentially having special importance as test beds for current theory. For more accurate properties, these EBs will likely need to be followed up both photometrically and spectroscopically. The brightness of these EBs will considerably facilitate their follow-up.

\subsection{Low-Mass EBs}

The first group consists of 11 low-mass EB candidates, including 10 newly discovered EBs with either $\mathrm{K}$ or M-dwarf stellar components. Our criteria for selecting these binaries were that they be well-detached, and that both components have estimated masses below $0.75 M_{\odot}$ (see Table 10 and Figure 21 ). Currently, only seven such detached low-mass EBs have been confirmed (YY Gem: Kron 1952; Torres \& Ribas 2002; CM Dra: Lacy 1977b; Metcalfe et al. 1996; CU Cnc: Delfosse et al. 1999; Ribas 2003; T-Her0-07621: Creevey et al. 2005; GU Boo: López-Morales \& Ribas 2005; NSVS01031772: López-Morales et al. 2006; and UNSW-TR-2: Young et al. 2006).

Despite a great deal of work that has been done to understand the structure of low-mass stars (e.g., Chabrier \& Baraffe 2000), models continue to underestimate their radii by as much as $15 \%$ (Lacy 1977a; Torres \& Ribas 2002; Creevey et al. 2005; Ribas 2006), a significant discrepancy considering that for solar-type stars the agreement with the observations is typically within 1-2\% (Andersen 1991, 1998). In recent years, an intriguing hypothesis has been put forward that strong magnetic fields may have bloated these stars through chromospheric activity (Ribas 2006; Torres et al. 2006; López-Morales 2007; Chabrier et al. 2007). Furthermore, Torres et al. (2006) find that such bloating occurs even for stars with nearly solar mass, and suggest that this effect may also be due to magnetically induced convective disruption. In either case, these radius discrepancies should diminish for widely separated binaries with long periods, as they become non-synchronous and thus rotate slower, which according to dynamo theory would reduce the strength of their magnetic fields.

Unfortunately, the small number of well-characterized lowmass EBs makes it difficult to provide strong observational constraints to theory. Despite the fact that such stars make up the majority of the Galactic stellar population, their intrinsic 
Table 9

EBs that Appear in Either the VSX or the SIMBAD Astronomical Databases

\begin{tabular}{|c|c|c|c|c|c|c|}
\hline Category & Object & $\alpha(\mathrm{J} 2000)$ & $\delta(\mathrm{J} 2000)$ & Spectral Type & Classification & Identifiers \\
\hline Circular & T-And0-00194 & 012012.816 & 483641.36 & A5 & Star & $\begin{array}{l}\text { BD+47 378; } \\
\text { GSC 03269-00662; } \\
\text { SAO 37126; AG+48 } 143 \\
\text { PPM 43886; } \\
\text { TYC 3269-662-1 }\end{array}$ \\
\hline Circular & T-And0-00459 & 011124.845 & 465749.44 & F8 & EB of Algol type & $\begin{array}{l}\text { CO And; GSC 03268-00398; } \\
\text { TYC 3268-398-1; BD+46 } \\
281 ; \text { BV } 74\end{array}$ \\
\hline Ambiguous & T-And0-00657 & 010606.159 & 473159.37 & K0 & Star & $\begin{array}{l}\text { BD+46 254; } \\
\text { GSC 03267-01349; } \\
\text { TYC 3267-1349-1 } \\
\text { AG+47 120; PPM } 43637\end{array}$ \\
\hline Circular & T-And0-00745 & 010345.076 & 445041.14 & & Star & $\begin{array}{l}\text { TYC 2811-470-1; } \\
\text { GSC 02811-00470 }\end{array}$ \\
\hline Ambiguous & T-And0-01203 & 010334.745 & 483239.27 & & Star & $\begin{array}{l}\text { TYC 3267-1176-1; } \\
\text { GSC 03267-01176 }\end{array}$ \\
\hline Circular & T-And0-04046 & 005520.157 & 474453.20 & & Star & GPM $13.833991+47.748193$ \\
\hline Roche-fill & T-And0-05153 & 011848.278 & 493936.86 & & EB of W UMa type & QW And \\
\hline Roche-fill & T-And0-05343 & 005255.122 & 480137.68 & & Star & GPM 13.232700+48.019757 \\
\hline Roche-fill & T-And0-07892 & 005615.567 & 483910.73 & & $\mathrm{~EB}$ & NSVS 3757820 \\
\hline Circular & T-And0-23792 & 005409.254 & 474519.91 & & Star & GPM $13.538629+47.755510$ \\
\hline Roche-fill & T-Cas0-00170 & 005337.847 & 484333.83 & & Star & $\begin{array}{l}\text { TYC 3266-195-1; } \\
\text { GSC 03266-00195 }\end{array}$ \\
\hline Eccentric & T-Cas0-00394 & 003251.608 & 491939.36 & B3 & EB of $\beta$ Lyr type & $\begin{array}{l}\text { V381 Cas; BD+48 162; } \\
\text { BV } 179\end{array}$ \\
\hline Roche-fill & T-Cas0-00430 & 004006.247 & 501415.64 & K4 & EB of W UMa type & $\begin{array}{l}\text { V523 Cas; } \\
\text { GSC 03257-00167; WR 16; } \\
\text { CSV 5867 } \\
\text { 1RXS J004005.0+501414; } \\
\text { TYC 3257-167-1 }\end{array}$ \\
\hline Circular & T-Cas0-00640 & 004706.277 & 483113.14 & & Star & $\begin{array}{l}\text { TYC 3266-765-1; } \\
\text { GSC 03266-00765 }\end{array}$ \\
\hline Circular & T-Cas0-00792 & 004826.554 & 513502.52 & & Star & $\begin{array}{l}\text { TYC 3274-664-1; } \\
\text { GSC 03274-00664 }\end{array}$ \\
\hline Roche-fill & T-Cas0-02013 & 004046.427 & 465657.41 & & Star & $\begin{array}{l}\text { TYC 3253-1767-1; } \\
\text { GSC 03253-01767 }\end{array}$ \\
\hline Inverted & T-Cas0-02069 & 004917.959 & 503902.92 & & $\mathrm{~EB}$ & V385 Cas \\
\hline Roche-fill & T-Cas0-08802 & 005132.351 & 471642.57 & & Star & GPM 12.884787+47.278540 \\
\hline Roche-fill & T-CrB0-00654 & 160014.507 & 351231.56 & & EB of W UMa type & $\begin{array}{l}\text { AS CrB; GSC 02579-01125; } \\
\text { NSVS } 7847829 \\
\text { ROTSE1 } \\
\text { J160014.54+351228.4 }\end{array}$ \\
\hline Roche-fill & T-CrB0-00705 & 155551.838 & 331100.39 & & EB of W UMa type & $\begin{array}{l}\text { ROTSE1 } \\
\mathrm{J} 155551.87+331100.5\end{array}$ \\
\hline Roche-fill & T-CrB0-01589 & 161009.313 & 355730.57 & & Variable of $\delta$ Sct type & $\begin{array}{l}\text { ROTSE1 } \\
\mathrm{J} 161009.33+355730.8\end{array}$ \\
\hline Roche-fill & T-CrB0-01605 & 160058.472 & 341854.34 & & EB of W UMa or RR Lyr-C & $\begin{array}{l}\text { NSVS 7848126; } \\
\text { ROTSE1 } \\
\text { J160058.45+341854.5 }\end{array}$ \\
\hline Roche-fill & T-CrB0-04254 & 160919.589 & 353211.48 & & EB of W UMa type & $\begin{array}{l}\text { ROTSE1 } \\
\text { J160919.62+353210.8 }\end{array}$ \\
\hline Circular & T-Cyg1-00246 & 194401.777 & 501357.42 & & Star & $\begin{array}{l}\text { TYC 3565-643-1; } \\
\text { GSC 03565-00643 }\end{array}$ \\
\hline Roche-fill & T-Cyg1-00402 & 195439.939 & 503641.91 & & Star & $\begin{array}{l}\text { TYC 3566-606-1; } \\
\text { GSC 03566-00606 }\end{array}$ \\
\hline Ambiguous & T-Cyg1-01385 & 201521.936 & 481714.14 & & Star & $\begin{array}{l}\text { TYC 3576-2035-1; } \\
\text { GSC 03576-02035 }\end{array}$ \\
\hline Circular & T-Cyg1-01627 & 194520.426 & 513507.22 & & Star & $\begin{array}{l}\text { TYC 3569-1752-1; } \\
\text { GSC 03569-01752 }\end{array}$ \\
\hline Roche-fill & T-Cyg1-04652 & 200707.305 & 503401.34 & & EB of W UMa type & GSC 03567-01035 \\
\hline Roche-fill & T-Cyg1-04852 & 195159.208 & 500529.61 & & EB of W UMa type & NSVS 5645908 \\
\hline Circular & T-Cyg1-09274 & 201606.814 & 515626.07 & & EB of W UMa type & $\begin{array}{l}\text { V1189 Cyg; CSV 8488; } \\
\text { GSC 03584-01600; SON } \\
7885\end{array}$ \\
\hline Roche-fill & T-Cyg1-11279 & 195953.377 & 492327.86 & & $\mathrm{X}$-ray source & 1RXS J195954.0+492318 \\
\hline
\end{tabular}




\begin{tabular}{|c|c|c|c|c|c|c|}
\hline Category & Object & $\alpha(\mathrm{J} 2000)$ & $\delta(\mathrm{J} 2000)$ & Spectral Type & Classification & Identifiers \\
\hline Roche-fill & T-Cyg1-14514 & 194805.077 & 525116.25 & & EB of W UMa or RR Lyr-C & $\begin{array}{l}\text { V997 Cyg; } \\
\text { GSC 03935-02233; ROTSE1 } \\
\text { J194804.79+525117.6; } \\
\text { SON } 7839\end{array}$ \\
\hline Ambiguous & T-Dra0-00240 & 170352.919 & 572155.54 & & Star & $\begin{array}{l}\text { TYC 3894-898-1; } \\
\text { GSC 03894-00898 }\end{array}$ \\
\hline Ambiguous & T-Dra0-00358 & 164538.339 & 543132.02 & & Star & $\begin{array}{l}\text { TYC 3879-2689-1; } \\
\text { GSC 03879-02689 }\end{array}$ \\
\hline Circular & T-Dra0-00398 & 165733.875 & 593151.98 & & EB of Algol type/X-ray source & $\begin{array}{l}\text { RX J1657.5+5931; 1RXS } \\
\text { J165733.5+593156 } \\
\text { VSX J165733.8+593151; } \\
\text { GSC 03898-00272 }\end{array}$ \\
\hline Roche-fill & T-Dra0-00405 & 162749.103 & 585023.30 & & Star & $\begin{array}{l}\text { TYC 3884-1488-1; } \\
\text { GSC 03884-01488 }\end{array}$ \\
\hline Roche-fill & T-Dra0-00959 & 162744.159 & 564559.30 & & EB of W UMa type/X-ray source & $\begin{array}{l}\text { NSVS } 2827877 ; 1 \text { RXS } \\
\text { J162743.9+564557 }\end{array}$ \\
\hline Circular & T-Dra0-01363 & 163420.417 & 570948.95 & $\mathrm{M} 4.5 \mathrm{~V}$ & $\begin{array}{l}\text { EB of BY Dra type } \\
\text { High proper-motion Star }\end{array}$ & $\begin{array}{l}\text { CM Dra; CSI+57-16335 1; } \\
\text { LSPM J1634+5709; } \\
\text { G 225-67; G 226-16 } \\
\text { IDS 16326+5721 A; } \\
\text { [RHG95] 2616; SBC7 580; } \\
\text { CCDM J16343+5710A } \\
\text { GJ 630.1 A; LP 101-15; IDS } \\
\text { 16325+5721 A; [GKL99] } \\
\text { 324; LHS 421 } \\
\text { 2MASS } \\
\text { J16342040+5709439; } \\
\text { CCABS 108; CABS 134; } \\
\text { GEN\# +9.80225067 } \\
\text { RX J1634.3+5709; 1RXH } \\
\text { J163421.2+570941; 1RXS } \\
\text { J163421.2+570933 } \\
\text { PM 16335+5715; USNO 168; } \\
\text { USNO-B1.0 1471-00307615; } \\
\text { NLTT 43148 }\end{array}$ \\
\hline Roche-fill & T-Dra0-01346 & 165212.345 & 574331.70 & & EB of Algol type & $\begin{array}{l}\text { BPS BS 16080-0095; } \\
\text { VSX J165212.3+574331; } \\
\text { GSC 03885-00583 }\end{array}$ \\
\hline Roche-fill & T-Dra0-02224 & 163001.408 & 544555.80 & & Star & BPS BS 16084-0159 \\
\hline Circular & T-Dra0-03021 & 170103.618 & 551454.70 & & EB of Algol type & $\begin{array}{l}\text { VSX J170103.5+551455; } \\
\text { GSC 03890-01216 }\end{array}$ \\
\hline Abnormal & T-Dra0-03105 & 162302.558 & 592723.44 & & X-ray source & 1RXS J162303.6+592717 \\
\hline Roche-fill & T-Dra0-05259 & 164148.751 & 562234.40 & & EB of W UMa type & $\begin{array}{l}\text { VSX J164148.7+562234; } \\
\text { GSC 03882-02264; } \\
\text { USNO-B1.0 1463-0278621 }\end{array}$ \\
\hline Ambiguous & T-Her0-00274 & 170051.150 & 452535.94 & & Star & $\begin{array}{l}\text { TYC 3501-2245-1; } \\
\text { GSC 03501-02245 }\end{array}$ \\
\hline Roche-fill & T-Her0-01086 & 164815.539 & 444428.73 & & EB of W UMa type & $\begin{array}{l}\text { GSC 03082-00896; } \\
\text { NSVS 5252572; } \\
\text { 1RXS J164817.3+4444430 }\end{array}$ \\
\hline Roche-fill & T-Her0-03579 & 163547.390 & 452458.19 & & EB of W UMa type & GSC 03499-01631 \\
\hline Inverted & T-Her0-05469 & 165451.245 & 432035.89 & & EB & V747 Her; SVS 2066 \\
\hline Circular & T-Lyr1-00359 & 191533.695 & 443701.30 & G0V & X-ray source & $\begin{array}{l}\text { V2277 Cyg; } \\
\text { GSC 03133-01149; ROTSE1 } \\
\text { J191533.92+443704.9 } \\
\text { BD+44 3087; ILF1+44 155; } \\
\text { 1RXS J191533.7+443704 }\end{array}$ \\
\hline Circular & T-Lyr1-00687 & 185527.911 & 471341.76 & & Star & $\begin{array}{l}\text { TYC 3544-1392-1; } \\
\text { GSC 03544-01392 }\end{array}$ \\
\hline Circular & T-Lyr1-01013 & 185503.963 & 474908.39 & & Star & $\begin{array}{l}\text { TYC 3544-2565-1; } \\
\text { GSC 03544-02565 }\end{array}$ \\
\hline Circular & T-Lyr1-01439 & 190613.439 & 465726.42 & & Star & $\begin{array}{l}\text { TYC 3545-2716-1; } \\
\text { GSC 03545-02716 }\end{array}$ \\
\hline Circular & T-Lyr1-02109 & 185735.415 & 450744.10 & & Cepheid variable star & $\begin{array}{l}\text { ROTSE1 } \\
\text { J185735.99+450752.5 }\end{array}$ \\
\hline Roche-fill & T-Lyr1-02166 & 190507.448 & 461507.51 & & $\mathrm{X}$-ray source & 1RXS J190504.8+461512 \\
\hline
\end{tabular}


Table 9

(Continued)

\begin{tabular}{|c|c|c|c|c|c|c|}
\hline Category & Object & $\alpha(\mathrm{J} 2000)$ & $\delta(\mathrm{J} 2000)$ & Spectral Type & Classification & Identifiers \\
\hline Roche-fill & T-Lyr1-03173 & 185945.531 & 472007.34 & & EB of W UMa type & ROTSE1 J185945.43+472007.0 \\
\hline Roche-fill & T-Lyr1-03211 & 184556.939 & 471909.54 & & EB of W UMa type/X-ray source & $\begin{array}{l}\text { ROTSE1 J184556.86+471914.4; } \\
\text { 1RXS J184557.9+471906 }\end{array}$ \\
\hline Roche-fill & T-Lyr1-03270 & 185733.098 & 480522.49 & & EB of W UMa type & ROTSE1 J185733.12+480522.5 \\
\hline Roche-fill & T-Lyr1-03783 & 185012.684 & 453544.05 & & Star & GPM $282.552858+45.595521$ \\
\hline Inverted & T-Lyr1-04431 & 191216.047 & 494223.58 & & EB of Algol type & $\begin{array}{l}\text { NSV 11822; } \\
\text { GSC 03550-01770; } \\
\text { NSVS 5578839; SON } 9371\end{array}$ \\
\hline Roche-fill & T-Lyr1-05706 & 184757.211 & 443811.30 & & EB of W UMa type & ROTSE1 J184757.18+443810.8 \\
\hline Inverted & T-Lyr1-05887 & 185210.489 & 474816.67 & & EB of Algol type & WX Dra; AN 24.1925 \\
\hline Roche-fill & T-Lyr1-06583 & 185226.837 & 445520.86 & & EB & ROTSE1 J185226.53+445527.8 \\
\hline Inverted & T-Lyr1-07179 & 184914.039 & 452438.61 & & Star & GPM $282.308454+45.410868$ \\
\hline Roche-fill & T-Lyr1-08406 & 185006.942 & 454105.95 & & Star & GPM $282.528833+45.685035$ \\
\hline Roche-fill & T-Lyr1-10276 & 184655.088 & 450052.27 & & EB of W UMa type & $\begin{array}{l}\text { V596 Lyr; } \\
\text { GPM 281.729421+45.014635; } \\
\text { GSC 03540-00085 } \\
\text { ROTSE1 J184654.98+450054.7 }\end{array}$ \\
\hline Inverted & T-Lyr1-10989 & 190622.791 & 454153.82 & & EB of Algol type & V512 Lyr; SON 10931 \\
\hline Roche-fill & T-Lyr1-11226 & 184521.748 & 455328.79 & & EB of W UMa type or $\delta$ Sct & $\begin{array}{l}\text { V594 Lyr; GPM } \\
\text { 281.340617+45.891326; GSC } \\
\text { 03540-01842 } \\
\text { ROTSE1 J184522.47+455321.0 }\end{array}$ \\
\hline Roche-fill & T-Lyr1-12772 & 185225.096 & 445540.23 & & EB of W UMa type & ROTSE1 J185226.53+445527.8 \\
\hline Abnormal & T-Lyr1-13166 & 190228.120 & 465857.75 & F9V & EB & V361 Lyr; SON 9349 \\
\hline Roche-fill & T-Per1-00328 & 034157.108 & 390729.60 & G5 & EB of Algol type & $\begin{array}{l}\text { HD 275743; BD+38 787; GSC } \\
02863-00755 ; \\
\text { TYC 2863-755-1 }\end{array}$ \\
\hline Circular & T-Per1-00459 & 033457.745 & 393318.70 & G5 & Star & $\begin{array}{l}\text { HD 275547; } \\
\text { GSC 02866-01995; } \\
\text { TYC 2866-1995-1 }\end{array}$ \\
\hline Circular & T-Per1-00750 & 034745.543 & 350037.08 & & Double or multiple star & $\begin{array}{l}\text { TYC 2364-2327-1; } \\
\text { GSC 02364-02327; } \\
\text { CCDM J03478+3501BC } \\
\text { ADS } 2771 \text { BC; BD+34 732B; } \\
\text { CSI+34 } 732 \text { 2; NSV } 1302\end{array}$ \\
\hline Roche-fill & T-Per1-00974 & 033443.738 & 384022.22 & A & Star & HD 275481 \\
\hline Circular & T-Per1-01218 & 034233.165 & 390603.63 & $\mathrm{~A}$ & $\mathrm{~EB}$ & $\begin{array}{l}\text { HU Per; HD 275742; } \\
\text { SVS } 922\end{array}$ \\
\hline Roche-fill & T-Per1-01482 & 034845.999 & 351410.05 & F0 & Star & HD 279025 \\
\hline Circular & T-Per1-02597 & 034432.202 & 395934.94 & $\mathrm{~K} 4 \mathrm{~V}$ & T Tau type Star & $\begin{array}{l}\text { [LH98] 94; } \\
\text { 1RXS J034432.1+395937; } \\
\text { 1SWASP J034433.95+395948.0 }\end{array}$ \\
\hline Inverted & T-Per1-04353 & 034504.887 & 374715.91 & & EB of Algol type & HV Per; SVS 368; P 107 \\
\hline Roche-fill & T-Tau0-00397 & 043009.466 & 253227.05 & A3 & EB of $\beta$ Lyr type & $\begin{array}{l}\text { GW Tau; SVS 1421; } \\
\text { HD 283709; } \\
\text { ASAS } 043009+2532.4\end{array}$ \\
\hline Inverted & T-Tau0-00686 & 040713.870 & 291832.44 & & EB of Algol type & IL Tau; SON 9543 \\
\hline Roche-fill & T-Tau0-00781 & 041251.218 & 244144.26 & G9 & Eruptive/T Tau-type Star & $\begin{array}{l}\text { V1198 Tau; NPM2+24.0013; } \\
\text { 1RXS J041250.9+244201 } \\
\text { GSC 01819-00498; } \\
\text { RX J0412.8+2442; [WKS96] } 14\end{array}$ \\
\hline Roche-fill & T-Tau0-01262 & 041628.109 & 280735.81 & K7V & Variable Star of Orion Type & $\begin{array}{l}\text { V1068 Tau; EM StHA 25; } \\
\text { JH 165; EM LkCa } 4 \\
\text { HBC } 370 ; \text { ASAS } 041628+2807.6\end{array}$ \\
\hline Roche-fill & T-Tau0-01715 & 041926.260 & 282614.30 & K7V & T Tau-type Star/X-ray source & $\begin{array}{l}\text { V819 Tau; HBC 378; } \\
\text { NAME WK X-Ray 1; } \\
\text { 1E 0416.3+2830 } \\
\text { IRAS C04162+2819; } \\
\text { TAP 27; [MWF83] P1; WK81 } 1 \\
\text { 1RXS J041926.1+282612; } \\
\text { X 04163+283 }\end{array}$ \\
\hline Roche-fill & T-Tau0-06463 & 040727.415 & 275106.36 & & EB of W UMa type & V1022 Tau; HV 6199; NSV 1464 \\
\hline
\end{tabular}


Table 9

(Continued)

\begin{tabular}{|c|c|c|c|c|c|c|}
\hline Category & Object & $\alpha(\mathrm{J} 2000)$ & $\delta(\mathrm{J} 2000)$ & Spectral Type & Classification & Identifiers \\
\hline Inverted & T-UMa0-00127 & 093806.716 & 560107.32 & $\mathrm{~A} 2 \mathrm{~V}$ & EB of Algol type & $\begin{array}{l}\text { VV UMa; GEN\# } \\
+0.05601395 ; \text { HIP } 47279 ; \\
\text { TYC 3810-1290-1 } \\
\text { GSC 03810-01290; SBC7 } \\
384 ; \text { GCRV 6211; BD+56 } \\
\text { 1395 } \\
\text { HIC } 47279 ; \text { SVS 770; } \\
\text { AAVSO 0931+56 }\end{array}$ \\
\hline Circular & T-UMa0-00222 & 100718.023 & 561237.12 & A0 & Star & $\begin{array}{l}\text { HD 237866; } \\
\text { GSC 03818-00504; SAO } \\
\text { 27524; AG+56 778; } \\
\text { HIC 49581 } \\
\text { BD+56 1432; HIP 49581; } \\
\text { YZ } 56 \text { 6209; TYC } \\
3818-504-1\end{array}$ \\
\hline Circular & T-UMa0-03090 & 100852.180 & 524552.49 & $\mathrm{~K} 2 \mathrm{e}$ & Star & $\begin{array}{l}\text { GSC 03815-01151; } \\
\text { RIXOS 229-302; } \\
\text { RX J100851.6+524553 }\end{array}$ \\
\hline Roche-fill & T-UMa0-03108 & 100416.780 & 541202.83 & & EB of W UMa type & NSVS 2532137 \\
\hline
\end{tabular}

Table 10

Low-mass EB Candidates $\left(M_{1,2}<0.75 M_{\odot}\right.$; Sorted by Mass $)$

\begin{tabular}{|c|c|c|c|c|c|c|c|c|c|c|}
\hline Category & Object & $\alpha(\mathrm{J} 2000)$ & $\delta(\mathrm{J} 2000)$ & Period (days) & $M_{1} / M_{\odot}$ & $M_{2} / M_{\odot}$ & Age (Gyr) & $\begin{array}{c}\text { Proper Motion } \\
\text { Source Catalog }\end{array}$ & $\begin{array}{c}\mathrm{PM}_{\alpha} \\
\left(\mathrm{mas} \mathrm{yr}^{-1}\right)\end{array}$ & $\begin{array}{c}\mathrm{PM}_{\delta} \\
\left(\mathrm{mas} \mathrm{yr}^{-1}\right)\end{array}$ \\
\hline Circular & T-Dra0-01363 ${ }^{\mathrm{b}}$ & 163420.417 & 570948.95 & 1.268 & $0.27 \pm 0.02$ & $0.24 \pm 0.03$ & $1.6 \pm 1.6$ & Salim \& Gould (2003) & -1121 & 1186 \\
\hline AmbigEq $^{c}$ & T-And0-10518 & 010744.417 & 484458.11 & 0.387 & $0.45 \pm 0.27$ & $0.45 \pm 0.28$ & $0.3 \pm 0.5$ & UCAC & 2.7 & -2.0 \\
\hline AmbigEq & T-Cyg1-12664 & 195139.824 & 481955.38 & 8.257 & $0.50 \pm 0.20$ & $0.48 \pm 0.19$ & $0.3 \pm 0.4$ & USNO-B & -18 & -6 \\
\hline AmbigEq & T-CrB0-14232 & 161022.495 & 335752.33 & 0.971 & $0.60 \pm 0.24$ & $0.55 \pm 0.29$ & $4.4 \pm 8.8$ & UCAC & -15.2 & -24.2 \\
\hline AmbigEq & T-CrB0-14543 & 155745.926 & 335607.28 & 1.506 & $0.60(-1)^{\mathrm{d}}$ & $0.60(-1)$ & $0.2(-2)$ & UCAC & -13.9 & 13.3 \\
\hline Circular & T-Per1-13685 & 035351.217 & 370316.73 & 0.384 & $0.60(-1)$ & $0.50(-1)$ & $10.0(-3)$ & UCAC & -24.1 & -15.9 \\
\hline AmbigEq & T-CrB0-10759 & 155218.455 & 303532.13 & 1.901 & $0.63 \pm 0.24$ & $0.62 \pm 0.21$ & $7.3 \pm 49.6$ & UCAC & 3.6 & -19.4 \\
\hline AmbigEq & T-UMa0-08238 & 100925.384 & 535701.31 & 1.250 & $0.69 \pm 0.54$ & $0.61 \pm 0.51$ & $4.1 \pm 15.0$ & USNO-B & 6 & -4 \\
\hline AmbigEq & T-Cas0-10450 & 002916.288 & 502738.58 & 8.656 & $0.71 \pm 0.21$ & $0.67 \pm 0.20$ & $0.3 \pm 0.4$ & UCAC & -3.1 & -4.2 \\
\hline AmbigEq & T-Dra0-07116 & 170253.025 & 550747.44 & 1.369 & $0.71 \pm 0.22$ & $0.69 \pm 0.22$ & $2.1 \pm 3.6$ & USNO-B & -2 & -16 \\
\hline Circular & T-Tau0-04859 & 040811.608 & 245110.18 & 3.068 & $0.74 \pm 0.10$ & $0.66 \pm 0.10$ & $8.8 \pm 14.8$ & UCAC & 3.4 & -8.0 \\
\hline
\end{tabular}

Notes.

${ }^{a}$ Where possible, we used the more accurate UCAC catalog, otherwise we reverted to the USNO-B catalog. Since they are dim and nearby, we expect most of the low-mass binaries to have comparably large proper motions.

b This binary is CM Draconis, which has been extensively studied and found to have a masses of $M_{1}=0.2307 \pm 0.0010 M_{\odot}$ and $M_{2}=0.2136 \pm 0.0010 M_{\odot}$ (Lacy 1977b; Metcalfe et al. 1996). For consistency, we listed the MECI results, which are off by less than $0.04 M_{\odot}(\sim 1.5 \sigma)$. We also adopted an alternative proper motion estimate, as its USNO-B values seems to be erroneous, probably due to its very high angular velocity.

${ }^{\mathrm{c}}$ For clarity we list for the ambiguous systems, only the solution with approximately equal components. But it is likely that at least a few of the ambiguous systems may be unequal, with half the period. Such cases can be identified as single-line spectroscopic binaries, with the secondary component being no larger than a few $0.1 M_{\odot}$.

${ }^{d}$ When the most likely model is at the edge of the parameter space, MECI is not able to bound the solution, and therefore cannot estimate the uncertainties. We mark $(-3)$ when the upper limit was reached, $(-2)$ when the lower limit was reached, and $(-1)$ if one of the other parameter is at its limit.

faintness renders them extremely rare objects in magnitudelimited surveys. In addition, once found, their low flux severely limits the ability to observe their spectra with both sufficiently high resolution and a high signal-to-noise ratio. To this end, the fact that the TrES survey was made with small-aperture telescopes is a great advantage, as any low-mass EB candidate found is guaranteed to be bright, and thus requires only moderateaperture telescopes for their follow-up. Thus we propose multiepoch spectroscopic study of the systems listed here, in order to confirm their low mass and to estimate their physical properties with an accuracy sufficient to test models of stellar structure. Moreover, two of our candidates (T-Cyg1-12664 and TCas0-10450), if they are in fact ambiguous-equal (group
[IV]), have periods greater than 8 days, making them prime targets for testing the aforementioned magnetic-bloating hypothesis.

\subsection{Eccentric EBs}

The second group of EBs consists of 34 binaries with eccentric orbits (see Table 3, and Figures 9-11). We were able to reliably measure values of $|e \cos \omega|$ as low as $\sim 0.005$ by using the eclipse timing technique (see Section 2 and Figure 3 ). Since this measure provides a lower limit to the eccentricity, it is well suited to identify eccentric EBs, even though the actual value of the eccentricity may be uncertain. As mentioned earlier, in an effort to avoid false-positives, we do 



Figure 17. LCs of abnormal EBs (panel 1).

not include in this group EBs whose eclipse timing measures $|e \cos \omega|<0.005$, or EBs with an eccentricity consistent with zero.

Our interest in these eccentric binaries stems from their potential to constrain tidal circularization theory (Darwin 1879). This theory describes how the eccentricity of a binary orbit decays over time due to tidal dissipation, with a characteristic timescale $\left(t_{\text {circ }}\right)$ that is a function of the components' stellar structure and orbital separation. As long as the components' stellar structure remains unchanged, the orbital eccentricity is expected to decay approximately exponentially over time $\left(e \propto \exp \left(-t / t_{\text {circ }}\right)\right)$. However, once the components evolve off the main sequence, this timescale may vary considerably (Zahn \& Bouchet 1989). Thus, to understand the circularization history of binaries with circularization timescales similar to or larger than their evolutionary timescales, one must integrate over the evolutionary tracks of both stellar components.
Three alternative tidal dissipation mechanisms have been proposed: dynamical tides (Zahn 1975, 1977), equilibrium tides (Zahn 1977; Hut 1981), and hydrodynamics (Tassoul 1988). Despite its long period of development, the inherent difficulty of observing tidal dissipation has prevented definitive conclusions. Zahn \& Bouchet (1989) add a further complication by maintaining that most of the orbital circularization process takes place at the beginning of the Hayashi phase, and that the eccentricity of a binary should then remain nearly constant throughout its lifetime on the main sequence.

Observational tests of these tidal circularization theories, whereby $t_{\text {circ }}$ is measured statistically in coeval stellar populations, have so far proved inconclusive. North \& Zahn (2003) found that short-period binaries in both the Large and Small Magellanic Clouds seem to have been circularized in agreement with the theory of dynamical tides. However, Meibom \& Mathieu (2005) show that, with the exception of the Hyades, the stars in the clusters that they observed were considerably 

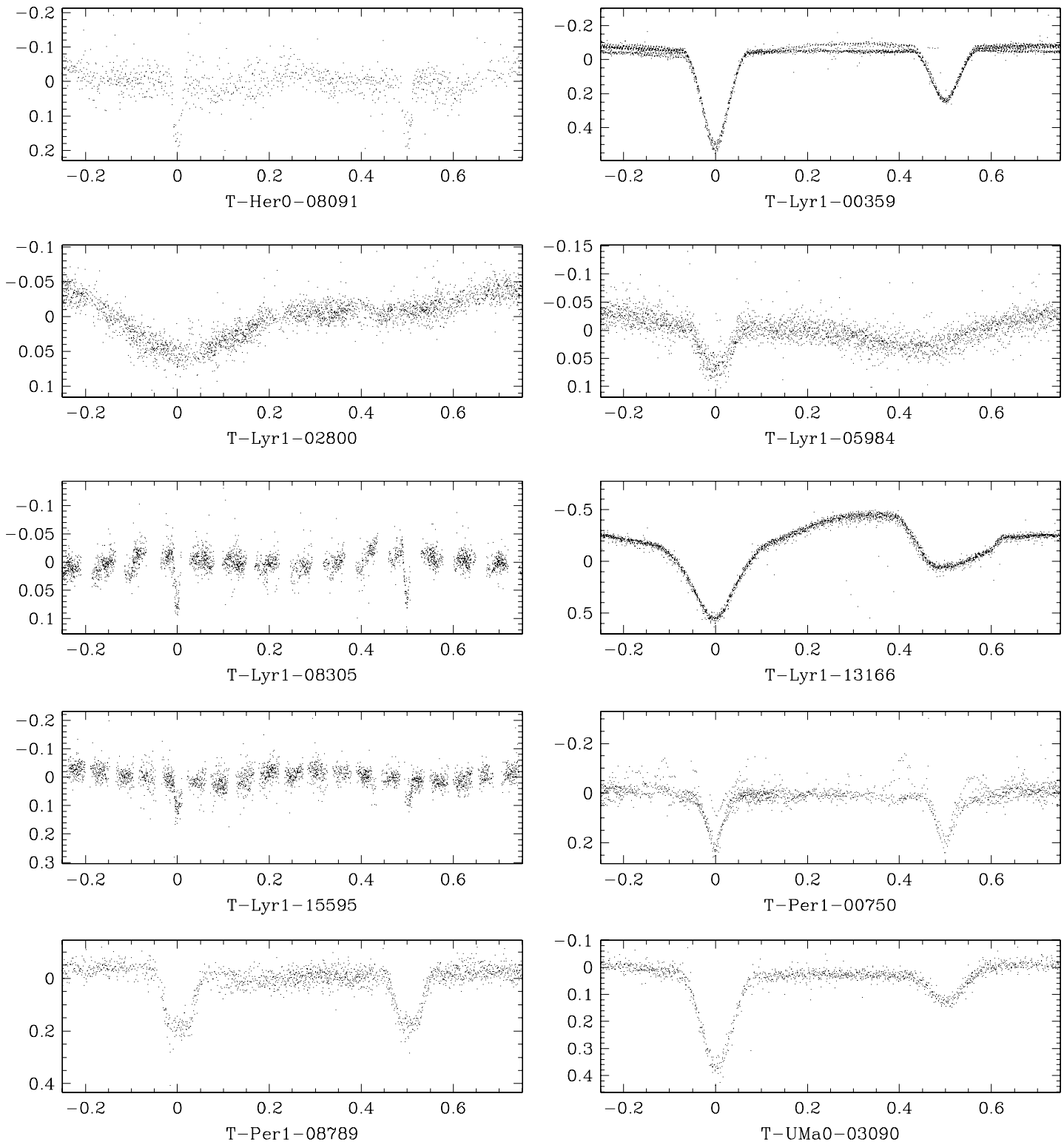

Figure 18. LCs of abnormal EBs (panel 2).

more circularized than any of the known dissipation mechanisms would predict. Furthermore, they find with a high degree of certainty, that older clusters are more circularized than younger ones, thereby contradicting the Hayashi phase circularization model.

Encouraged by the statistical effect of circularization that can be seen in our catalog (Figure 22), we further estimated $t_{\text {circ }}$ for each of the eccentric systems as follows. Zahn $(1977,1978)$ provides an estimate for the orbital circularization timescale due to turbulent dissipation in stars possessing a convective envelope, assuming that corotation has been achieved:

$$
t_{\text {circ }}=\frac{1}{21 q(1+q) k_{2}}\left(\frac{M R^{2}}{L}\right)^{1 / 3}\left(\frac{a}{R}\right)^{8}
$$

where $M, R, L$ are the star's mass, radius, and luminosity, and $k_{2}$ is the apsidal motion constant of the star, which is determined by its internal structure and dynamics.
More massive stars, which do not have a convective envelope but rather develop a radiative envelope, are thought to circularize their orbit using radiative damping (Zahn 1975; Claret \& Cunha 1997). This is a far slower mechanism, whose circularization timescale can be estimated by

$$
t_{\text {circ }}=\frac{2}{21 q(1+q)^{11 / 6} E_{2}}\left(\frac{R^{3}}{G M}\right)^{1 / 2}\left(\frac{a}{R}\right)^{21 / 2}
$$

where $E_{2}$ is the tidal torque constant of the star, and $G$ is the universal gravitational constant. We can greatly simplify these expressions by applying Kepler's law $\left(a^{3}=\operatorname{GM}(1+q)(P / 2 \pi)^{2}\right)$, and adopt the Cox (2000) power-law approximations for the main-sequence mass-radius and mass-luminosity relations. For the convective envelope case, we adopt the late-type massradius relation $\left(M<1.3 M_{\odot}\right)$, and for the radiative envelope case we adopt the early-type mass-radius relation 




Figure 19. The mass-mass relation for the detached EBs of the TrES dataset. Each category is represented by a different symbol. Note that the ambiguous EBs are plotted twice, where only one of the solutions can be correct. Note also that the equal-component solutions are clustered along the diagonal, while the unequal-component solutions are clustered along the minimum available mass of the Yonsei-Yale isochrones $\left(0.4 M_{\odot}\right)$. Some of the ambiguous solutions deviate from these clusters due to poor constraints on the secondary eclipse, which brings about a large uncertainty. Finally, note the sparsity of EBs populating the lowmass corner of this plot $\left(M_{1,2}<0.75 M_{\odot}\right)$. These systems, whose importance is outlined in Section 3.1, were modeled using the Baraffe isochrones. CM Draconis (T-Dra0-01363) clearly sets itself apart, being the lowest-mass binary in the catalog (circle at bottom left).

(A color version of this figure is available in the online journal)

$\left(M \geqslant 1.3 M_{\odot}\right)$, thus arriving at

$$
t_{\text {circ }} \simeq\left\{\begin{array}{c}
0.53 \operatorname{Myr}\left(k_{2} / 0.005\right)^{-1} q^{-1}(1+q)^{5 / 3}(P / \text { day })^{16 / 3} \\
\quad \times\left(M / M_{\odot}\right)^{-4.99}, M<1.3 M_{\odot} \\
1370 \operatorname{Myr}\left(E_{2} / 10^{-8}\right)^{-1} q^{-1}(1+q)^{5 / 3}(P / \text { day })^{7} \\
\quad \times\left(M / M_{\odot}\right)^{-2.76}, M \geqslant 1.3 M_{\odot} .
\end{array}\right.
$$

Determining the values of $k_{2}$ and $E_{2}$ is the most difficult part of this exercise, since their values are a function of the detailed structure and dynamics of the given star, which in turn changes significantly as the star evolves (Claret and Cunha 1997; Claret and Willems 2002). In our calculation, we estimate these values by interpolating published theoretical tables ( $k_{2}$ : Zahn 1994, $E_{2}$ : Zahn 1975; Claret and Cunha 1997). Since both stellar components contribute to the circularization process, the combined circularization timescale becomes $t_{\text {circ }}=$ $1 /\left(t_{\text {circ }, 1}^{-1}+t_{\text {circ }, 2}^{-1}\right)$, where the subscripts 1 and 2 refer to the primary and secondary binary components (Claret and Cunha 1997). In Table 3, we list the combined circularization timescale for each of the eccentric EBs we identify.

The value of $t_{\text {circ }}$ for most of the eccentric systems (21 of 34 ) is larger than the Hubble time, indicating that no significant circularization is expected to have taken place since they settled on the main-sequence. About a quarter of the eccentric systems ( 8 of 34) have a $t_{\text {circ }}$ smaller than the Hubble time but larger than 1 Gyr. While circularization is underway, the fact that they are still eccentric is consistent with theoretical expectations. The remaining systems ( 5 of 34) all have $t_{\text {circ }}<1 \mathrm{Gyr}$, have periods less than 3.3 days, and unless they are extremely young, require an explanation for their eccentric orbits. Two of these EBs (T-Tau0-02487 and T-Tau0-03916) are located near the

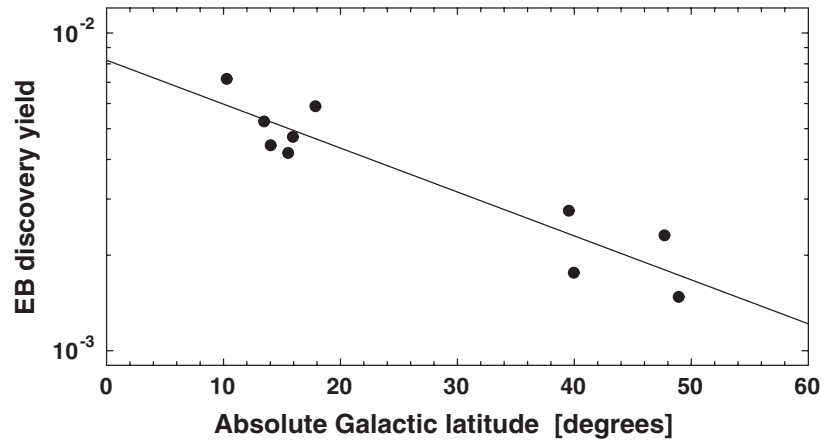

Figure 20. The relation between the EB discovery yield (the fraction of LCs found to be EBs) and the absolute value of the Galactic latitude, or $|b|$, for the ten TrES fields used in this catalog (see Tables 1 and 2). The solid line is the linear regression of the $\log$ of the EB discovery yield $\left(r^{2}=0.867\right)$. Some of the residual scatter can be explained as being due to differences in the duration of observations in each field. By including the duration in a bi-linear regression, we get a substantially improved fit $\left(r^{2}=0.911\right)$.

star-forming regions of Taurus, supporting the hypothesis that they are indeed young. However, this hypothesis does not seem to be adequate for T-Cas0-02603, which has a period of only 2.2 days and $t_{\text {circ }} \simeq 0.26 \mathrm{Gyr}$, while possessing a large eccentricity of $e \simeq 0.25$. An alternative explanation is that some of these binaries were once further apart, having larger orbital periods, and thus larger circularization timescales. These systems may have been involved in a comparably recent interaction with a third star (a collision or near miss), or have been influenced by repeated resonant perturbations of a tertiary companion.

Finally, we would like to draw the reader's attention to our shortest-period eccentric EB, T-Cas0-00394, whose period is a mere 1.7 days. Notably, this system is entirely consistent with theory, since its mass falls in a precarious gap, where the stellar envelopes of its components are no longer convective, yet their radiative envelopes are not sufficiently extended to produce significant tidal drag (see Figure 23).

\subsection{Abnormal EBs}

The third group of EBs consists of 20 abnormal systems (see Table 5, and Figures 17 and 18). While possessing the distinctive characteristics of EBs, these LCs stood out during manual inspection for a variety of reasons. These systems underline the difficulty of fully automating any LC pipeline, as any such system will inevitably need to recognize atypical EBs that were not encountered before.

The LCs we listed can be loosely classified into groups according to the way they deviate from a simple EB model. A few cases exhibited pulsation-like fluctuations that were not synchronized with the EB period (shorter-period: T-Dra0-00398, longer-period: T-Lyr1-00359, T-Per1-00750). These fluctuations may be due either to the activity of an EB component, or to a third star whose light is blended with the binary. In principle, one can identify the active star by examining the amplitude of the fluctuations during the eclipses. If the fluctuations originate from one of the components, their observed amplitude will be reduced when the component is being eclipsed. In such a case, if the fluctuations are due to pulsations, they can further provide independent constraints to the stellar properties through astroseismological models (Mkrtichian et al. 2004). To identify such fluctuating EBs one must subtract the fitted EB model from the LC, and evaluate the residuals (e.g., Pilecki \& Szczygiel 2007). When the fluctuation period is fixed, one can simply search the 

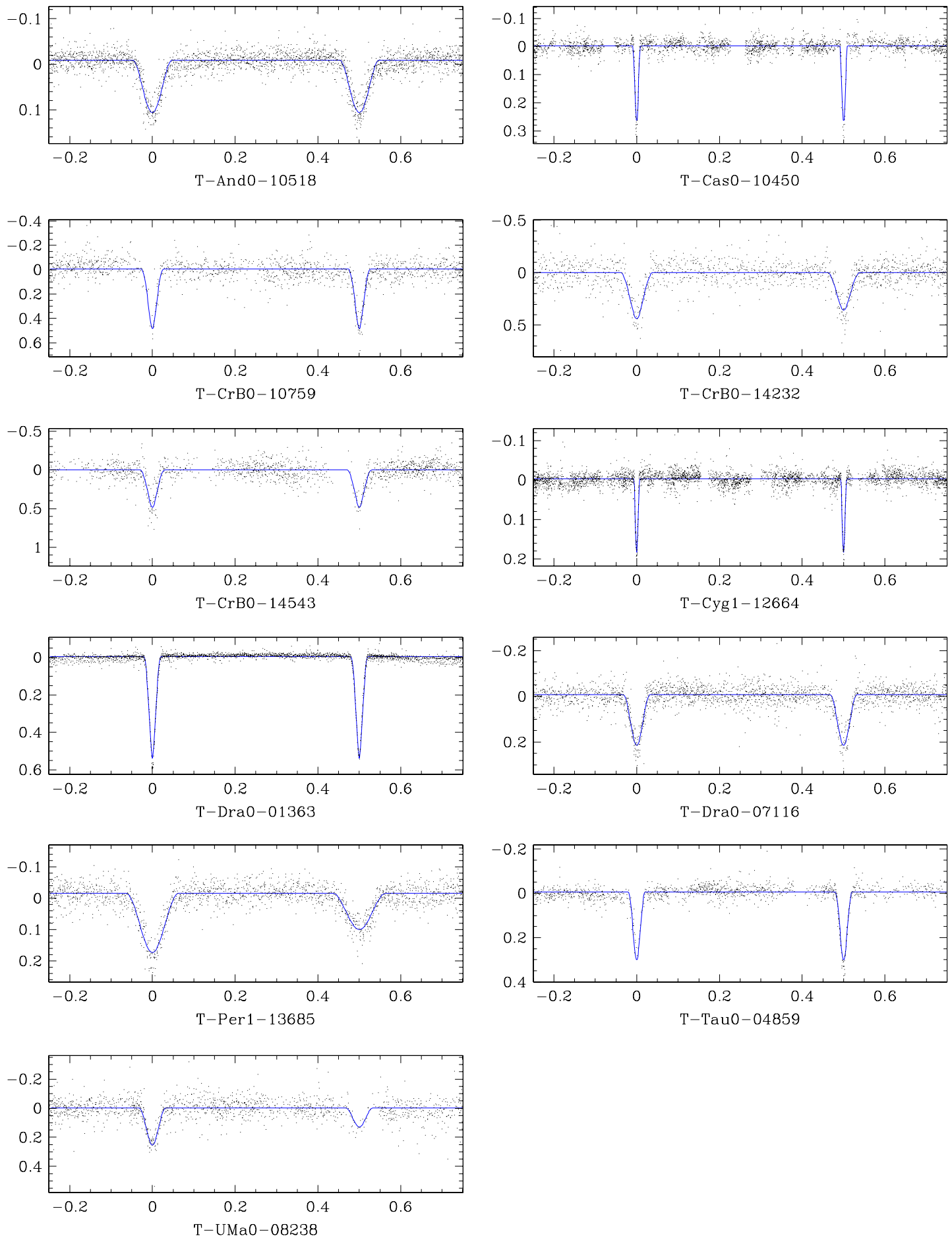

Figure 21. Low-mass candidates $\left(M_{1}<0.75 M_{\odot}\right)$, with their best-fit MECI models (solid line).

(A color version of this figure is available in the online journal)

residual LC using a periodogram, as was done in step (1) of our pipeline (see Section 2). However, when the fluctuation period varies (i.e. non-coherent), as in the aforementioned LCs, one must employ alternative methods, since simply phasing their LC will not produce any discernable structure. For LCs with long-period fluctuations, one can directly search the residuals for time dependencies, while for LCs with short-period fluctuations one can search the residuals for non-Gaussian distribu- tions. However, in practice these measurements will likely not be robust, as there are many instrumental effects that can produce false positives. Thus, we employ a search for auto-correlations in the residual time series, which overcomes most instrumental effects, while providing a reliable indicator for many types of pseudo-periodic fluctuations.

The remaining systems had LC distortions that appear to be synchronized with the orbital period. The source of these 


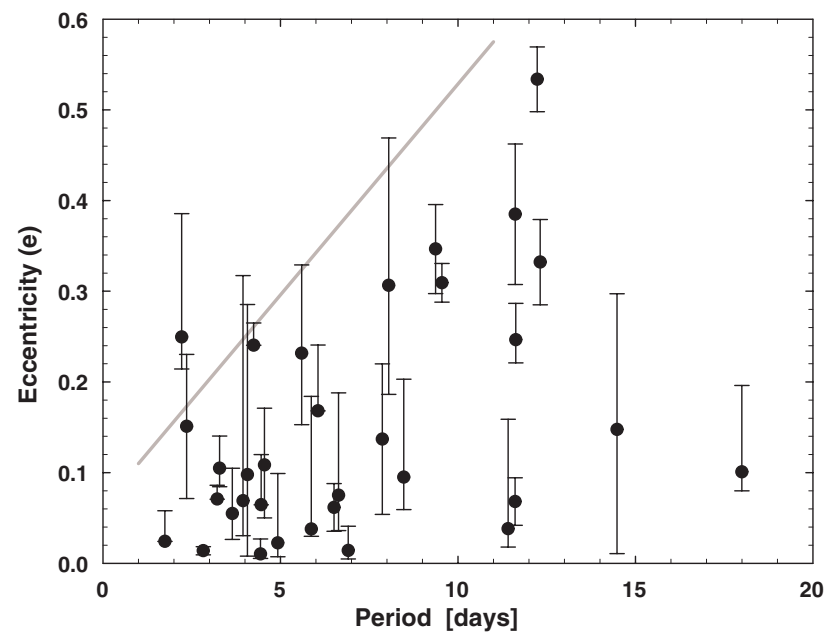

Figure 22. The period-eccentricity relation. The lower ends of the error bars were truncated, where needed, by the measured lower limit, $|e \cos \omega|$. Note the lack of eccentric short-period systems. The diagonal line is provided to guide the eye.

fluctuations is likely due to long-lasting surface inhomogeneities on one or both of the rotationally synchronized components. When the LC has brief periodic episodes of darkening (T-And011476, T-Cas0-13944, T-Cyg1-07584, T-Dra0-04520), they can usually be explained as stable star spots, but brief periodic episodes of brightening (T-And0-04594, T-Her0-08091), which may indicate the presence of stable hot-spots, are more difficult to interpret. This phenomenon is especially puzzling in the aforementioned two cases, in which the brightening episodes are briefer than one would expect from a persistent surface feature and repeat at the middle of both plateaux.

When the two plateaux of an LC are not flat, they are usually symmetric about the center of the eclipses. This is due to the physical mirror symmetry about the line intersecting the binary components' centers. When the axis of symmetry does not coincide with the center of eclipse (T-And0-00920, T-Cyg1-08866, T-Dra0-03105, T-Lyr1-07584, T-Lyr1-15595), a phenomenon we term "eclipse offset," we conclude that this symmetry must somehow be broken. This may occur if the EB components are not rotationally synchronized, or have a substantial tidal lag. Another form of this asymmetry can appear as an amplitude difference between the two LC plateaux (T-Her0-03497, T-Lyr1-13166, T-Per1-08789, T-UMa0-03090). This phenomenon, which was originally called the "periastron effect" and has since been renamed the "O'Connell effect," has been known for over a century, and has been extensively studied (e.g., O'Connell 1951; Milone 1986). Classic hypotheses suggest an uneven distribution of circumstellar material orbiting with the binary (Struve 1948) or surrounding the stars (Mergentaler 1950), either of which could induce a preferential $H^{-}$absorption on one side. Binnendijk (1960) was the first of many to suggest that this asymmetry is due to subluminous regions of the stellar surface (i.e. star spots). However, this explanation also requires the stars to be rotationally synchronized, and for the spots to be stable over the duration of the observations. Alternative models abound, including a hot spot on one side of a component brought about through mass transfer from the other component, persistent star spots created by an off-axis magnetic field, and circumstellar material being captured by the components and heating one side of both stars (Liu \& Yang 2003). As with many phenomena that have multiple possible

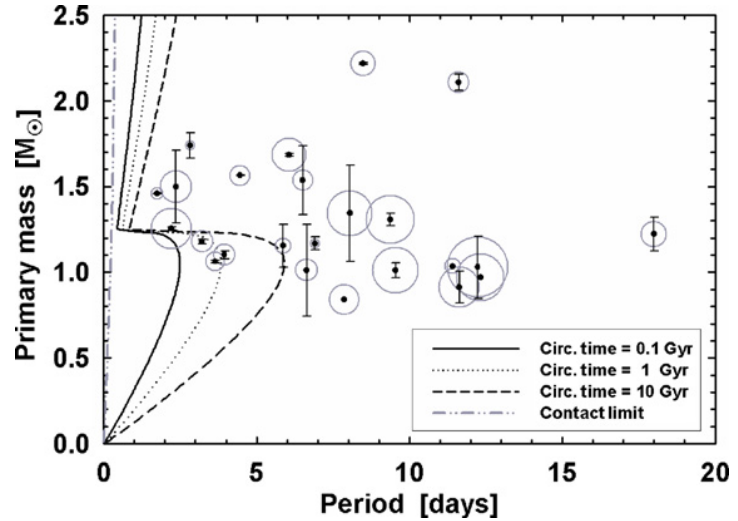

Figure 23. The period-primary mass relation for eccentric EBs. We included all systems with well-determined masses. The area of the gray circles is proportional to the EB's eccentricity. All the curves are theoretical boundaries, assuming that the binary components are both on the main sequence and have equal masses $(q=1)$. The left-most dot-dash line demarcates the binary contact limit, and the remaining curves mark systems with increasing circularization time (see Equation (10)). Note the abrupt increase in the circularization time for systems more massive than $\sim 1.25 M_{\odot}$, at which point the stellar convective envelope becomes radiative, and thus far less efficient at tidal dissipation.

(A color version of this figure is available in the online journal)

models, the true answer may involve a combination of a number of these mechanisms, and will likely vary from system to system (Davidge \& Milone 1984).

Finally, a few particularly unusual LCs (T-Dra0-03105, TLyr1-05984) display a very large difference between their eclipse durations. Although a moderate difference could be explained by an eccentric orbit, such extreme eccentricities in systems with such short orbital periods ( 0.5 and 1.5 days) are highly unlikely.

\section{CONCLUSIONS}

We presented a catalog of 773 eclipsing binaries found in ten fields of the TrES survey, identified and analyzed using an automated pipeline. We described the pipeline we used to identify and model them. The pipeline was designed to be mostly automated, with manual inspections taking place only once the vast majority of non-EB LCs had been automatically filtered out. At the final stage of the pipeline, we classified the EBs into seven groups: eccentric, circular, ambiguous-equal, ambiguousunequal, inverted, Roche-lobe-filling, and abnormal. The former four groups were all successfully modeled with our model fitting program. However, the latter three groups possessed significant additional physical phenomena (tidal distortions, mass-transfer, and surface activity), which did not conform to the simple detached-EB model we employed.

We highlighted three groups of binaries, which may be of particular interest and warrant follow-up observations. These groups are low-mass EBs, EBs with eccentric orbits, and abnormal EBs. The low-mass EBs (both components $<0.75 M_{\odot}$ ) allow one to probe the mass-radius relation at the bottom of the main-sequence. Only seven such EBs have previously been confirmed, and the physical properties of many of them are inconsistent with current theoretical models. Our group of ten new candidates will likely provide considerable additional constraints to the models, and the discovery of two long-period systems could help confirm a recent hypothesis that this inconsistency is due to stellar magnetic activity. The eccentric-orbit EBs may help confirm and constrain tidal circularization theory, as 
many of them have comparably short circularization timescales. We demonstrated that, as one would predict from the theory, the shortest-period systems fall within a narrow range of masses, in which their stellar envelopes cease to be convective yet their envelopes are not extended enough to produce significant tidal drag. The abnormal EBs seem to show a plethora of effects that are indicative of asymmetries, stellar activity, persistent hot and cold spots, and a host of other physical phenomena. Some of these systems may require dedicated study to be properly understood.

In the future, as LC datasets continue to grow, it will become increasingly necessary to use such automated pipelines to identify rare and interesting targets. Such systematic searches promise a wealth of data that can be used to test and constrain theories in regions of their parameter space that were previously inaccessible. Furthermore, even once the physics of "vanilla" systems has been solved, more complex cases will emerge to challenge us to achieve a better understanding of how stars form, evolve, and interact.

We would like to thank Tsevi Mazeh for many useful discussions, as well as Søren Meibom for his repeated help. We would also like to thank Sarah Dykstra for her continuous support throughout the preparation of this paper. We are grateful to the staff of the Palomar Observatory for their assistance in operating the Sleuth instrument, and we acknowledge support from NASA through grant NNG05GJ29G issued through the Origins of Solar Systems Program. This research has made use of the NASA's Astrophysics Data System Bibliographic Services, the SIMBAD database, operated at CDS, Strasbourg, and the VSX database, which was created by Christopher Watson for the AAVSO. This publication also utilizes data products from 2MASS, which is a joint project of the University of Massachusetts and the Infrared Processing and Analysis Center/California Institute of Technology, funded by NASA and NSF. Finally, we would like to thank the anonymous referee for very insightful comments and suggestions, which significantly improved this manuscript.

\section{APPENDIX A}

\section{REJECTING SINGLE-ECLIPSE EB MODELS}

An EB LC, comprising a deep eclipse and a very shallow eclipse, can occur in one of two ways. Either the secondary component is luminous but extremely small (e.g., a white dwarf observed in UV), thus producing a shallow primary eclipse, or the secondary component is comparably large but extremely dim, thus producing a shallow secondary eclipse. The first case, though possible (e.g., Maxted et al. 2004), is extremely rare, and will have a signature "flat bottom" to the eclipse. We have not encountered such an LC in our dataset. The second case will have a rounded eclipse bottom, due to the primary component's limb darkening. Assuming this latter contingency, in which the secondary component is dark in comparison to the primary component, we can place a lower bound on its radius $\left(R_{2}\right)$ :

$$
R_{2} \geqslant R_{1} \sqrt{1-10^{-0.4 \Delta \mathrm{mag}_{1}}}
$$

where $R_{1}$ is the radius of the primary component, and $\Delta$ mag $_{1}$ is the magnitude depth of the primary eclipse. Thus, if the eclipse is very deep, the size of the secondary component must approach the size of the primary component. However, coeval short-period detached EBs with components of similar sizes yet desperate luminosities are expected to be very rare, assuming that they follow normal stellar evolution. Therefore, if only one eclipse is detected, and it is both rounded and sufficiently deep, we may conclude that this configuration entry is likely to be incorrect, and that the correct configuration has double the orbital period and produces two equal eclipses. Only when we cannot apply such a period-doubling solution (i.e. when the secondary eclipse is detectable) do we resort to questioning our assumption of normal stellar evolution (see classification group $\mathrm{V}$, described in Section 2).

\section{APPENDIX B}

\section{DESCRIPTION OF THE CATALOG FIELDS}

Due to the large size of the catalog, we were only able to list small excerpts of it in the body of this paper. Readers interested in viewing the catalog in its entirety can download it electronically. Note that although the catalog lists 773 unique systems, each of the 103 ambiguous EBs appears in both possible configurations (see Section 2), raising the total number of catalog entries to 876. Below, we briefly describe the catalog's 38 columns. The column units, if any, are listed in square brackets.

1. Category - the EB's classification (see Section 2).

2. Binary name - the EB's designation, which is composed of its TrES field (see Table 1) and index.

3. $\alpha$-the EB's right ascension (J2000).

4. $\delta$-the EB's declination (J2000).

5. Period (days) — the EB's orbital period.

6. Period uncertainty (days) - the uncertainty in the EB's orbital period.

7. $\operatorname{Mass}_{1}\left(M_{\odot}\right)$-the mass of the EB's primary (more massive) component.

8. Mass ${ }_{1}$ uncertainty $\left(M_{\odot}\right)$ - the uncertainty in the primary component's mass.

9. $\operatorname{Mass}_{2}\left(M_{\odot}\right)$ - the mass of the EB's secondary (less massive) component.

10. Mass 2 uncertainty $\left(M_{\odot}\right)$ - the uncertainty in the secondary component's mass.

11. Age (Gyr) - the age of the EB (assumed to be coeval).

12. Age uncertainty (Gyr) - the uncertainty in the EB's age.

13. Score - a weighted reduced $\chi^{2}$ of the MECI model fit (see Devor \& Charbonneau 2006b for further details).

14. Isochrone source-isochrone tables used (Y2: Kim et al. 2002, or Baraffe: Baraffe et al. 1998).

15. Color weighting - the relative weight $(w)$ of the LC fit, compared to the color fit (see Devor \& Charbonneau 2006b for further details).

16. $P M$ source - the database that provided the proper motion measurement (UCAC: Zacharias et al. 2004, USNO-B: Monet et al. 2003, or Salim03: Salim \& Gould 2003).

17. $P M_{\alpha}$ (mas $\left.\mathrm{yr}^{-1}\right)$ - the right ascension component of the EB's proper motion.

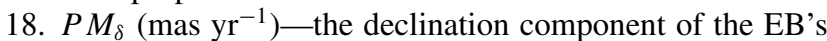
proper motion.

19. Location error (arcsec) - the distance between our listed location (columns 3 and 4) and the location listed by the proper motion database.

20. $\operatorname{mag}_{B}$ - the USNO-B $B$-band observational magnitude of the EB (average of both magnitude measurements, if available). 
21. mag $_{R}$ - the USNO-B $R$-band observational magnitude of the EB (average of both magnitude measurements, if available).

22. Third-light fraction - the fraction of third-light flux $(R$ band) blended into the LC (i.e. the flux within 30", excluding the target, divided by the total flux within $30^{\prime \prime}$ ).

23. mag $_{J}$ - the 2MASS observational $J$-band magnitude of the EB, converted to ESO $J$-band.

24. mag $_{H}$ - the 2MASS observational $H$-band magnitude of the EB, converted to ESO $H$-band.

25. mag $_{K}$ - the 2MASS observational $K_{S}$-band magnitude of the EB, converted to ESO $K$-band.

26. $\mathrm{Mag}_{J}$ - the absolute ESO $J$-band magnitude of the EB listed in the isochrone tables.

27. $\mathrm{Mag}_{H}$ - the absolute ESO $H$-band magnitude of the EB listed in the isochrone tables.

28. $\mathrm{Mag}_{K}$ - the absolute ESO $\mathrm{K}$-band magnitude of the EB listed in the isochrone tables.

29. Distance (pc) - the distance to the EB, as calculated from the extinction-corrected distance modulus.

30. $A(V)$ - the EB's V-mag absorption due to Galactic interstellar extinction (assuming $R_{V}=3.1$ ).

31. $\sin (i)$ - the sine of the EB's orbital inclination.

32. $|e \cos (\omega)|-$ a robust lower limit for the EB's eccentricity (see Equation (1)).

33. Eccentricity - the orbital eccentricity of the EB.

34. Eccentricity uncertainty - the uncertainty in the orbital eccentricity of the EB.

35. $\Delta$ mag $_{1}$ - the $r$-band primary (deeper) eclipse depth in magnitudes.

36. Epoch $_{1}$ - the Heliocentric Julian date (HJD) at the center of a primary eclipse, minus $2,400,000$.

37. $\Delta \mathrm{mag}_{2}$ — the $r$-band secondary (shallower) eclipse depth in magnitudes.

38. Epoch $_{2}$ - the Heliocentric Julian date (HJD) at the center of a secondary eclipse, minus 2,400,000.

Note that the values of the uncertainties (columns 6, 810 , 12 , and 34) were calculated by measuring the curvature of the parameter-space $\chi^{2}$ contour, near its minimum. This method implicitly assumes a Gaussian distribution of the parameter likelihood. If the likelihood distribution not Gaussian, but rather has a flattened (boxy) distribution, then the computed uncertainty becomes large. In extreme cases, the estimated formal uncertainty can be larger than the measurement itself.

\section{REFERENCES}

Agol, E., Steffen, J., Sari, R., \& Clarkson, W. 2005, MNRAS, 359, 567

Akerlof, C., et al. 2000, AJ, 119, 1901

Alard, C., \& Guibert, J. 1997, A\&A, 326, 1

Alcock, C., et al. 1998, ApJ, 492, 190

Alonso, R., et al. 2004, ApJ, 613, L153

Andersen, J. 1991, A\&A Rev., 3, 91

Andersen, J. 1998, IAU Symp. 189, Fundamental Stellar Properties (Paris: IAU), 99

Baglin, A., \& The COROT Team 1998, IAU Symp. 185, New Eyes to See Inside the Sun and Stars (Paris: IAU), 301

Bakos, G., Noyes, R. W., Kovács, G., Stanek, K. Z., Sasselov, D. D., \& Domsa, I. 2004, PASP, 116, 266

Baraffe, I., Chabrier, G., Allard, F., \& Hauschildt, P. H. 1998, A\&A, 337, 403

Beaulieu, J. P., et al. 1995, A\&A, 299, 168

Binnendijk, L. 1960, AJ, 65, 358

Borucki, W. J., Koch, D. G., Dunham, E. W., \& Jenkins, J. M. 1997, Planets Beyond the Solar System and the Next Generation of Space Missions, in ASP Conf. Ser. 119, ed. D. Soderblom (San Francisco: ASP), 153

Carpenter, J. M. 2001, AJ, 121, 2851
Chabrier, G., \& Baraffe, I. 2000, ARA\&A, 38, 337

Chabrier, G., Gallardo, J., \& Baraffe, I. 2007, arXiv:0707.1792

Charbonneau, D., Brown, T. M., Latham, D. W., \& Mayor, M. 2000, ApJ, 529, L45

Charbonneau, D., Brown, T. M., Burrows, A., \& Laughlin, G. 2007, in Protostars and Planets V, ed. B. Reipurth, D. Jewitt, \& K. Keil (Tucson, AZ: University of Arizona Press), 701-716

Christian, D. J., et al. 2006, MNRAS, 372, 1117

Claret, A. 1998, A\&A, 335, 647

Claret, A. 2000, A\&A, 363, 1081

Claret, A., \& Cunha, N. C. S. 1997, A\&A, 318, 187

Claret, A., \& Willems, B. 2002, A\&A, 388, 518

Crawford, J. A. 1955, ApJ, 121, 71

Creevey, O. L., et al. 2005, ApJ, 625, L127

Cox, A. N. 2000, Allen's Astrophysical Quantities (4th ed.; New York: AIP)

Darwin, G. H. 1879, The Observatory, 3, 79

Davidge, T. J., \& Milone, E. F. 1984, ApJS, 55, 571

Deeg, H. J., Doyle, L. R., Kozhevnikov, V. P., Blue, J. E., Martín, E. L., \& Schneider, J. 2000, A\&A, 358, L5

Delfosse, X., Forveille, T., Mayor, M., Burnet, M., \& Perrier, C. 1999, A\&A, 341, L63

Derekas, A., Kiss, L. L., \& Bedding, T. R. 2007, arXiv astro-ph/0703137

Devor, J. 2005, ApJ, 628, 411

Devor, J., \& Charbonneau, D. 2006a, Ap\&SS, 304, 351

Devor, J., \& Charbonneau, D. 2006b, ApJ, 653, 648

Dunham, E. W., Mandushev, G. I., Taylor, B. W., \& Oetiker, B. 2004, PASP, 116,1072

Eggleton, P. P. 1983, ApJ, 268, 368

Etzel, P. B. 1991, IAPP Commun., 45, 25

Gilmore, G., et al. 1998, Proc. SPIE Conf., 3350, 541

Gorda, S. Y., \& Svechnikov, M. A. 1998, Astron. Rep., 42, 793

Henry, G. W., Marcy, G. W., Butler, R. P., \& Vogt, S. S. 2000, ApJ, 529, L41

Holman, M. J., \& Murray, N. W. 2005, Science, 307, 1288

Hut, P. 1981, A\&A, 99, 126

Iben, I., Jr., \& Tutukov, A. V. 1984, ApJS, 54, 335

Kaiser, N., et al. 2002, Proc. SPIE, 4836, 154

Kim, Y., Demarque, P., Yi, S. K., \& Alexander, D. R. 2002, ApJS, 143,499

Kirkpatrick, S., Gelatt, C. D., \& Vecchi, M. P. 1983, Science, 220, 671

Kovács, G., Zucker, S., \& Mazeh, T. 2002, A\&A, 391, 369

Kron, G. E. 1952, ApJ, 115, 301

Kukarkin, B. V., \& Parengo, P. P. 1948, General Catalogue of Variable Stars (Moscow: Nauka)

Kurucz, R. L. 1992, IAU Symp. 149, The Stellar Populations of Galaxies (Paris: IAU), 225

Lacy, C. H. 1977a, ApJ, 218, 444

Lacy, C. H. 1977b, ApJS, 34, 479

Liu, Q.-Y., \& Yang, Y.-L. 2003, Chin. J. Astron. Astrophys., 3, 142

López-Morales, M., \& Ribas, I. 2005, ApJ, 631, 1120

López-Morales, M., Orosz, J. A., Shaw, J. S., Havelka, L., Arevalo, M. J., McIntyre, T., \& Lazaro, C. 2006, arXiv astro-ph/0610225

López-Morales, M. 2007, ApJ, 660, 732

Mandushev, G., et al. 2005, ApJ, 621, 1061

Maxted, P. F. L., Marsh, T. R., Morales-Rueda, L., Barstow, M. A., Dobbie, P. D., Schreiber, M. R., Dhillon, V. S., \& Brinkworth, C. S. 2004, MNRAS, 355,1143

Mazeh, T., et al. 2000, ApJ, 532, L55

Mazeh, T., Tamuz, O., \& North, P. 2006, MNRAS, 367, 1531

McCullough, P. R., et al. 2006, ApJ, 648, 1228

Meibom, S., \& Mathieu, R. D. 2005, ApJ, 620, 970

Mergentaler, J. 1950, Wroclaw Cont., 4,

Metcalfe, T. S., Mathieu, R. D., Latham, D. W., \& Torres, G. 1996, ApJ, 456,356

Milone, E. F. 1986, ApJS, 61, 455

Mkrtichian, D. E., et al. 2004, A\&A, 419, 1015

Monet, D. G., et al. 2003, AJ, 125, 984

Nelder, J. A., \& Mead, R. 1965, Comput. J., 7, 308

North, P., \& Zahn, J.-P. 2003, A\&A, 405, 677

O'Connell, D. J. K. 1951, Publ. Riverview Coll. Obs., 2, 85

O'Donovan, F. T., Charbonneau, D., \& Kotredes, L. 2004, The Search for Other Worlds, Vol. 713 (New York: AIP), 169

O'Donovan, F. T., et al. 2006, ApJ, 644, 1237

O’Donovan, F. T., et al. 2007, ApJ, 662, 658

Paczynski, B. 1986, ApJ, 304, 1 
Paczynski, B. 1997, The Extragalactic Distance Scale, in Space Telescope Science Institute Ser., ed. M. Livio (Cambridge: Cambridge University Press), 273-280

Pilecki, B., \& Szczygiel, D. M. 2007, Inform. Bull. Variable Stars, 5768,1

Pojmanski, G. 1997, Acta Astron., 47, 467

Popper, D. M., \& Etzel, P. B. 1981, AJ, 86, 102

Press, W. H., Teukolsky, S. A., Vetterling, W. T., \& Flannery, B. P. 1992, Numerical Recipes in C: The Art of Scientific Computing (2nd ed.; Cambridge: Cambridge University Press)

Ribas, I. 2003, A\&A, 398, 239

Ribas, I. 2006, Ap\&SS, 304, 87

Salim, S., \& Gould, A. 2003, ApJ, 582, 1011

Samus, N. N. 2006, Astron. Astrophys. Trans., 25, 223

Schwarzenberg-Czerny, A. 1989, MNRAS, 241, 153

Schwarzenberg-Czerny, A. 1996, ApJ, 460, L107

Skrutskie, M. F., et al. 2006, AJ, 131, 1163

Stebbing, J. 1910, ApJ, 32, 185

Struve, O. 1948, PASP, 60, 160

Tamuz, O., Mazeh, T., \& North, P. 2006, MNRAS, 367, 1521
Tassoul, J.-L. 1988, ApJ, 324, L71

Torres, G., Lacy, C. H., Marschall, L. A., Sheets, H. A., \& Mader, J. A. 2006, ApJ, 640, 1018

Torres, G., \& Ribas, I. 2002, ApJ, 567, 1140

Tsesevich, V. P. 1973, Eclipsing Variable Stars (New York: Wiley)

Tyson, J. A. 2002, Proc. SPIE, 4836, 10

Udalski, A. 2003, Acta Astron., 53, 291

Udalski, A., et al. 1994, Acta Astron., 44, 165

Wilson, R. E., \& Devinney, E. J. 1971, ApJ, 166, 605

Yi, S., Demarque, P., Kim, Y.-C., Lee, Y.-W., Ree, C. H., Lejeune, T., \& Barnes, S. 2001, ApJS, 136, 417

Young, T. B., Hidas, M. G., Webb, J. K., Ashley, M. C. B., Christiansen, J. L. Derekas, A., \& Nutto, C. 2006, MNRAS, 370, 1529

Zacharias, N., Urban, S. E., Zacharias, M. I., Wycoff, G. L., Hall, D. M., Monet, D. G., \& Rafferty, T. J. 2004, AJ, 127, 3043

Zahn, J.-P. 1975, A\&A, 41, 329

Zahn, J.-P. 1977, A\&A, 57, 383

Zahn, J.-P. 1978, A\&A, 67, 162

Zahn, J.-P., \& Bouchet, L. 1989, A\&A, 223, 112

Zahn, J.-P. 1994, A\&A, 288, 829 